\title{
ISOMETRIC ACTIONS ON SPHERES WITH AN ORBIFOLD QUOTIENT
}

\author{
CLAUDIO GORODSKI AND ALEXANDER LYTCHAK
}

\begin{abstract}
We classify representations of compact connected Lie groups whose induced action on the unit sphere has an orbit space isometric to a Riemannian orbifold.
\end{abstract}

\section{INTRODUCTION}

In this paper we classify all orthogonal representations of compact connected groups $G$ on Euclidean unit spheres $\mathbb{S}^{n}$ for which the quotient $\mathbb{S}^{n} / G$ is a Riemannian orbifold. We are going to call such representations infinitesimally polar, since they can be equivalently defined by the condition that slice representations at all non-zero vectors are polar. The interest in such representations is two-fold.

On the one hand, one can hope to isolate an interesting class of representations in this way. This class contains all polar representations, which can be defined by the property that the corresponding quotient space $\mathbb{S}^{n} / G$ is an orbifold of constant curvature 1 . Polar representations very often play an important and special role in geometric questions concerning representations (cf. [PT88, BCO03]), and the class investigated in this paper consists of closest relatives of polar representations.

On the other hand, any such quotient has positive curvature and all geodesics in the quotient are closed. Any of these two properties is extremely interesting and in both classes the number of known manifold examples is very limited (cf. [Zil12, Bes78]). We have hoped to obtain new examples of positively curved manifolds and of manifolds with closed geodesics as universal orbi-covering of such spaces. Should the orbifolds be bad one could still hope to obtain new examples of interesting orbifolds. For instance, the two-dimensional weighted complex projective spaces are very special examples of rotationally invariant singular Zoll surfaces ([Bes78, chapter 4] and [GUW09]). And the four-dimensional weighted quaternionic projective space $\mathbb{S}^{7} / \mathbf{S U}(2)$, where $\mathbf{S U}(2)$ acts by the irreducible representation of complex dimension 4, is the so called Hitchin orbifold $O_{3}$ (cf. [Zil07] and the references therein). This orbifold with a different positively curved metric is the starting point of the only new positively curved manifold discovered in the last decades [Dea11, GVZ11]. We have hoped that new interesting examples of orbifolds may arise in this way.

Unfortunately, this hope was not fulfilled. Our main result shows that no interesting new examples occur. Namely, we prove:

Theorem 1.1. Let $\rho$ be an orthogonal representation of a compact Lie group $G$ on a Euclidean space $\mathbb{R}^{n+1}$. Assume that the quotient $X=\mathbb{S}^{n} / G$ is a Riemannian orbifold. Then the universal orbi-covering $\tilde{X}$ of $X$ is either a weighted complex or a weighted quaternionic projective space, or $\tilde{X}$ has constant curvature 1 or 4.

Date: September 3, 2018.

The first author was partially supported by the CNPq grant 303038/2013-6 and the FAPESP project $2011 / 21362-2$. 
As mentioned above, $\tilde{X}$ has constant curvature 1 if and only if the action of $G$ is polar, a very well understood situation [Dad85]. It turns out that $\tilde{X}$ is a weighted projective space if and only if it has dimension 2 or $G$ acts almost freely and has rank 1, again two very well known cases [Str94, GG88.

Almost free actions and polar representations provide orbifolds of arbitrary dimension. On the other hand, the remaining case of quotients of constant curvature 4 can occur only in low cohomogeneity, maybe the most surprising consequence of our classification.

Theorem 1.2. Let $X=\mathbb{S}^{n} / G$ be a Riemannian orbifold. If $G$ has rank at least 2 and the representation is not polar, then the dimension $k$ of $X$ satisfies $2 \leq k \leq 5$.

This result becomes more surprising if one compares it with the world of non-homogeneous singular Riemannian foliations on spheres. Recent examples of M. Radeschi [Rad14 show that there are singular Riemannian foliations on round spheres $\mathbb{S}^{n}$, for $n$ large enough, such that the quotient space is isometric to a hemisphere of curvature 4, which can be of arbitrary large dimension!

On the other hand, for more standard examples of infinitesimally polar actions the differences are not that big. For polar foliations, it is known that the only quotients that arise, arise as quotients of group representations [Tho00. Moreover, in higher codimensions essentially all polar foliations are homogeneous [Tho91]. The case of $G$ having rank one and acting almost freely corresponds to the case of regular Riemannian foliations. All such foliations but the 7-dimensional Hopf fibration are homogeneous ([LW13] and the literature therein). We refer also to $\operatorname{Rad12}$ for a related result.

As it turns out, there are only very few representations satisfying the assumptions of Theorem 1.2 for $k \geq 3$, the case $k=2$ having been previously classified in [Str94]. At least if we restrict the representation to the connected component, all such representations are of a similar type: they are, up to a circle factor, the double of a standard vector representation. In the Table 1 and further in the text we will use the following notation: by $\mathbb{S}^{k}(r), \mathbb{S}_{+}^{k}(r)$, $\mathbb{S}_{++}^{k}(r), \mathbb{S}_{+++}^{k}(r)$ we denote the round sphere of constant curvature $\frac{1}{r^{2}}$ quotiented by the group $\Gamma$ which is respectively generated by $0,1,2,3$ commuting reflections. We call the corresponding spaces the sphere, hemisphere, quarter-sphere and eighth-sphere of curvature $\frac{1}{r^{2}}$, respectively.

Theorem 1.3. Let $\rho: G \rightarrow \mathbf{S O}(n+1)$ be an orthogonal representation of a compact connected Lie group $G$ such that the quotient space $X=\mathbb{S}^{n} / G$ is a Riemannian orbifold of dimension $k$. Assume that $\rho$ is non-polar, $G$ has rank at least 2 and $k \geq 3$. Then $\rho$ is one of the following:

\begin{tabular}{|c|c|c|c|}
\hline$G$ & $\rho$ & Conditions & Orbit space \\
\hline $\mathbf{S p i n}(9)$ & $\mathbb{R}^{16} \oplus \mathbb{R}^{16}$ & - & $\mathbb{S}_{++}^{3}\left(\frac{1}{2}\right)$ \\
$\mathbf{S U}(n)$ & $\mathbb{C}^{n} \oplus \mathbb{C}^{n}$ & $n \geq 3$ & $\mathbb{S}_{+}^{3}\left(\frac{1}{2}\right)$ \\
$\mathbf{U}(n)$ & $\mathbb{C}^{n} \oplus \mathbb{C}^{n}$ & $n \geq 2$ & $\mathbb{S}_{+}^{3}\left(\frac{1}{2}\right)$ \\
$\mathbf{S p}(n)$ & $\mathbb{H}^{n} \oplus \mathbb{H}^{n}$ & $n \geq 2$ & $\mathbb{S}_{+}^{5}\left(\frac{1}{2}\right)$ \\
$\mathbf{S p}(n) \mathbf{U}(1)$ & $\mathbb{C}^{2 n} \oplus \mathbb{C}^{2 n}$ & $n \geq 2$ & $\mathbb{S}_{++}^{4}\left(\frac{1}{2}\right)$ \\
$\mathbf{S p}(n) \mathbf{S p}(1)$ & $\mathbb{R}^{4 n} \oplus \mathbb{R}^{4 n}$ & $n \geq 2$ & $\mathbb{S}_{++}^{3}\left(\frac{1}{2}\right)$ \\
$\mathbf{T}^{2} \times \mathbf{S p}(n)$ & $\mathbb{C}^{2 n} \oplus \mathbb{C}^{2 n}$ & $n \geq 2$ & $\mathbb{S}_{+++}^{3}\left(\frac{1}{2}\right)$ \\
\hline
\end{tabular}

TABle 1: QuOTIENTS OF CONSTANT CURVATURE 4 
We can now summarize our classification:

Theorem 1.4. Let a compact Lie group $G$ act effectively and isometrically on the unit sphere $\mathbb{S}^{n}$. Let $\rho: G^{0} \rightarrow \mathbf{S O}(n+1)$ be the corresponding representation of the identity component. The quotient $\mathbb{S}^{n} / G$ is a Riemannian orbifold if and only if one of the following cases occur for $\rho$ :

- The representation $\rho$ is polar;

- $G^{0}=S^{1}$ and $\rho$ is a sum of non-trivial irreducible representations;

- $G^{0}=\mathbf{S U}(2)$ and $\rho$ is a sum of non-trivial irreducible quaternionic representations;

- $\rho$ has cohomogeneity 3 ;

- $\rho$ is described in Theorem 1.3.

All good quotient orbifolds appearing in Theorem 1.4 have curvatures between 1 and 4 and all bad quotient orbifolds appearing in Theorem 1.4 have diameter $\pi / 2$, hence curvature $\leq 4$ at some points. We are not aware of a geometric reason, why the curvature cannot be constant equal to 9, or just larger than 4 at every tangent plane. Due to [LT10], for any representation $\rho: G \rightarrow \mathbf{S O}(n+1)$ the quotient $X=\mathbb{S}^{n} / G$ always has regular points with arbitrary large curvatures at some plane, unless $X$ is a Riemannian orbifold, hence described above. On the other hand, it seems to be an interesting question, how large the infimum of the curvatures can be in a general quotient space $X=\mathbb{S}^{n} / G$. We hope to discuss this question closely related to [MS05, Gre00] in a forthcoming paper.

Many parts of our proof apply to non-homogeneous singular Riemannian foliations. Some observations may be of independent interest, for instance the following:

Theorem 1.5. Let $X$ be a compact positively curved Riemannian orbifold of dimension at least 3. If $X$ has non-empty boundary as an Alexandrov space, then $X$ is a good orbifold. Moreover, the universal orbi-covering of $X$ is diffeomorphic to a sphere.

In dimension 2 the above theorem is not true: a two-dimensional disc with a singularity at the boundary (half of a tear-drop orbifold) is a bad orbifold and can be equipped with a positively curved metric. Indeed, this orbifold arises as the orbit space of certain isometric group actions on unit spheres (compare Example 5.1). In view of this result, [FGT12, Theorem 2.10] and [FG14], it seems reasonable to ask the following:

Question 1.1. Do all orbifolds from Theorem 1.5 carry a metric of constant curvature?

Another observation that may be of independent interest in other contexts is a reduction procedure similar to the reduction of the principal isotropy group [LR79, Str94, GS00, GL14]. Namely, we show in Section 5 that one can view any stratum $S$ in a given quotient space $M / G$ as an open subset of a (usually non-injective) isometric immersion of another quotient space $F / N$, where $F$ is a closed and totally geodesic submanifold of $M$ and $N$ a subquotient group of $G$. In the particular case in which $S$ is a closed stratum, this amounts to the easy statement that the whole manifold $S$ is isometric to some quotient $F / N$ as above. In our opinion, it is exactly this point, that does not have a similar for general singular Riemannian foliations, which is responsible for the great variety of examples in Rad14. For instance (cf. [Rad14, Subsection 6.2]), assume for the moment that our quotient $\mathbb{S}^{n} / G$ is a hemi-sphere of constant curvature greater than 1 . Then the boundary sphere $S$ is a closed stratum of our quotient and thus, by the observation above, the manifold $S$ is itself isometric to the quotient of another sphere, $S=\mathbb{S}^{l} / N$. But then $N$ must have rank one and $S$ must be $\mathbb{C} P^{1}$ or $\mathbb{H} P^{1}$ hence of dimension 2 or 4 . If a similar reduction procedure existed for general 
singular Riemannian foliations, we would get a fibration $\mathbb{S}^{l} \rightarrow S$, and the dimension of $S$ would be 2, 4 or 8 . However, according to [Rad14], the dimension of $S$ can be an arbitrary number.

This work has the following structure. In Section 2 we collect basics about general quotient spaces and Riemannian orbifolds. In the last part of the section we explain that the usual reduction of the principal isotropy group may be used to reduce the classification of possible quotient orbifolds $\mathbb{S}^{n} / G$ to the case in which all isotropy groups of $G$ are products of groups of rank one, with very special slice representations (Lemma 2.3). It would have been possible to use this information together with some algebraic tools (cf. [Sch80, How95]) to prove Theorem 1.4 essentially by means of representation theory only, however in a rather elaborate way. We choose a different, more geometric path. In Section 3 we discuss the geometry of all the quotient spaces appearing in Theorem 1.4, in particular, verifying the last column of Table 1. In Section 4 we first discuss some general facts about Coxeter orbifolds, and describe in Lemma 4.3 under which conditions they are good orbifolds. Then we use convexity arguments from the Soul Theorem and the theorem of Fraenkel-Petrunin to prove Theorem 1.5. Already at this point we know that topologically no interesting new manifolds can occur in our classification. In Section 5, we use the reduction procedure described above to begin with the investigation of the structure of possible quotient spaces $\mathbb{S}^{n} / G$ isometric to a $k$-dimensional Riemannian orbifold $X$, with $k \geq 3$. Under the assumption that $X$ has non-empty boundary, i.e., that $X$ is not a weighted projective space, we apply the reduction and find another spherical quotient $\mathbb{S}^{m} / N$, which is a Riemannian orbifold $Y$ of dimension $(k-1)$. Moreover, the action of $N$ is non-polar if the action of $G$ is non-polar. In the last section we use this as the main inductive step in our argument. Before embarking on the proof of the main result, we discuss in Section 6 a special case. In Proposition 6.1 we discuss which sums of two representations of cohomogeneity 1 satisfy the assumption of Theorem 1.4, obtaining exactly the representations listed in Table 1. In Section 7 we prove by induction on the dimension $k$ of the quotient $\mathbb{S}^{n} / G$ a central result, which is the last piece of information that we need to finally deduce in Section 8 the remaining claims. Note, that by the general strata-reduction argument from Section 5 it suffices to prove the results for $k \leq 6$. In principle, one could apply brutal force and write down all representations of cohomogeneity $\leq 7$. Our proof is more geometric: we use the inductive structure to show that the representation can be reducible only as a sum of two representations of cohomogeneity 1, which finishes the reducible case, due to the Section 6. Finally, we use again the inductive structure to reduce the number of possible irreducible candidates to very few ones, which are then excluded case-by-case.

For the sake of convenience, we generally assume that our representations are almost effective.

Acknowledgment. The second named author would like to thank Gudlaugur Thorbergsson for very constructive discussions at the time of the work on [LT10], during which the idea for the present paper was born.

\section{Preliminaries}

2.1. Strata of isometric group actions. Let $G$ be a closed group of isometries of a complete connected Riemannian manifold $M$. Let $X$ denote the quotient metric space $M / G$. The manifold $M$ is stratified by $G$-invariant submanifolds consisting of points whose isotropy groups are conjugate to each other. The projection of any such stratum to $X$ is called an 
isotropy stratum of $X$. Any isotropy stratum is an (often non-complete and disconnected) Riemannian manifold, which is a locally convex subset of the Alexandrov space $X$. Any closure of any isotropy stratum is an extremal subset of the Alexandrov space $X$ (cf. Pet07, section 4.1]). From the geometric point of view, the more natural stratification of $X$ consists of the connected components of isotropy strata defined above. These components (which also coincide with the components of normal isotropy strata [Sch80, Introduction]) can also be defined as the connected components of the set of points in $X$ with isometric tangent cones. Hence, they are determined uniquely by the metric space $X$. In particular, they do not depend on the presentation of the metric space $X$ as a quotient $X=M / G$. We will call them the metric strata or, simply, the strata of $X$. Referring to the general structure of Alexandrov spaces, these closures are exactly the primitive extremal subsets of $X$, and thus correspond to the canonical metric stratification of the Alexandrov space $X$ Pet07, Section 4.1(8)].

There is a unique full-dimensional isotropy stratum of $X$, corresponding to the minimal isotropy group, called the principal isotropy group. This stratum is always connected, convex, open and dense in $X$. It is called the principal stratum and consists of all principal orbits of the $G$-action.

We would like to mention, that the structure described above answers Question 4.6(2) in AKLM03.

2.2. Polar representations. We refer to [PT88, Tho00] for accounts on polar representations. Recall that a representation $\rho: G \rightarrow \mathbf{O}(n+1)$ is polar if and only the restriction of the representation to the identity component is orbit-equivalent to the isotropy representation of a symmetric space. This happens if and only if the quotient space $\mathbb{R}^{n+1} / G$ is a flat Riemannian orbifold. An equivalent property is that the quotient $\mathbb{S}^{n} / G$ of the corresponding unit sphere is a Riemannian orbifold of constant curvature 1.

2.3. Riemannian orbifolds. We assume some experience with orbifolds. We refer the reader to [Dav10] and [KL11] for the basic background. Some information can also be found in [GL14, section 3] and much more information in [BH99], pp. 584-618.

A Riemannian orbifold comes along with a canonical stratification. Each stratum is a connected Riemannian manifold, which is locally convex with respect to the ambient metric. The closure of any stratum is a union of strata. Any Riemannian orbifold $X$ can be written as a quotient of a Riemannian manifold (the orthonormal frame bundle of $X$ ) under an almost free isometric action of a compact Lie group. The canonical stratification of $X$ is then exactly the stratification described in Subsection 2.1. The boundary of the orbifold (in the sense of Alexandrov geometry) is the closure of the union of strata of codimension 1.

Any Riemannian orbifold $X$ has a unique universal orbi-covering $\tilde{X}$ with a discrete isometric action of $\Gamma=\pi_{1}^{\text {orb }}(X)$ on $\tilde{X}$. The orbifold $X$ has a non-empty boundary if and only if $\Gamma$ contains a reflection, i.e., an involution with the set of fixed points of codimension 1 in $\tilde{X}$.

2.4. Orbifolds as quotients. We recall from [LT10] that for an isometric action of a compact Lie group $G$ on a Riemannian manifold $M$, the quotient $X=M / G$ is a Riemannian orbifold if and only if all slice representations of the action are polar. In [LT10] an isometric action of $G$ on $M$ with this property has been called infinitesimally polar. Thus an orthogonal representation is infinitesimally polar in the sense of this paper if and only if the corresponding action on the unit sphere is infinitesimally polar in the sense of [LT10]. 
An isometric action of a compact Lie group $G$ on a Riemannian manifold $M$ is infinitesimally polar if and only it is the case for the restriction of the action to the identity component $G^{0}$ of $G$. In such a case the canonical projection $M / G^{0} \rightarrow M / G$ is an orbi-covering.

Assuming again that the quotient $X=M / G$ is a Riemannian orbifold, all singular orbits of the action are contained in boundary of $X$. Moreover, if $\pi_{1}(M)=1$ and $G$ is connected then the boundary of $X$ is exactly the set of singular $G$-orbits, while its complement is the union of principal and exceptional orbits [Lyt10]. In particular, $X$ has no boundary if and only the action of $G$ has no singular orbits.

Assume now that $M$ has positive curvature. If the principal isotropy group of the action of $G$ on $M$ is non-trivial then the quotient $X=M / G$ has non-empty boundary (Wil06], Lemma 5 and subsequent lines). Hence, if $X$ is a Riemannian orbifold and has empty boundary, the action of $G$ on $M$ must be almost free.

Recall now that any action of a $k$-dimensional torus on a compact positively curved manifold always has at least one orbit of dimension at most 1, thus with an isotropy group of rank at least $(k-1)$ Wil03, Lemma 6.1]. Hence:

Lemma 2.1. Let a compact Lie group $G$ act by isometries on a compact positively curved Riemannian manifold $M$. If the quotient space $M / G$ has empty boundary and it is isometric to a Riemannian orbifold of positive dimension then $G$ has rank 1 and the action is almost free.

2.5. Quotient-geodesics. Let $M$ be a connected complete Riemannian manifold on which a compact Lie group $G$ acts by isometries with quotient space $X=M / G$. A geodesic in $M$ is called $G$-horizontal, if it is orthogonal to the $G$-orbits it meets. A geodesic is horizontal if and only if it starts in a horizontal direction. The projection to $X$ of a horizontal geodesic is a concatenation of metric geodesics (i.e. locally distance minimizing curves) in $X$. We call such a projection a quotient-geodesic. We refer to [LT10, Section 4] for more on this subject and for proofs of further statements below.

The projections of two $G$-horizontal geodesics coincide if they coincide initially. Hence, we can uniquely extend an initial piece of a quotient-geodesic to a quotient-geodesic defined on $\mathbb{R}$. Since any metric geodesic in $X$ is a quotient-geodesic in the above sense, we thus can uniquely extend any geodesic to an infinite quotient-geodesic. The set of quotient-geodesics is closed under point-wise convergence.

Remark 2.1. In fact, any quotient-geodesic is a so-called quasi-geodesic of the Alexandrov space $X$ ([Pet07, Section 5]), a notion which will not play a role below. Unlike general quasi-geodesics, the quotient geodesics are uniquely defined by their initial segments. We have a well defined continuous quotient-geodesic flow on $X$.

There is a dense subset in the space of quotient-geodesics that run only through the principal stratum and strata of codimension 1. This union $X_{1}$ of all strata of codimension at most 1 is a Riemannian orbifold. Moreover, the quotient-geodesics contained in $X_{1}$ coincide with the orbifold-geodesics in the Riemannian orbifold $X_{1}$. By the above density claim, the quotient-geodesics in $X$ are limits of orbifold-geodesics in $X_{1}$, hence they are defined only in metric terms, independently of the presentation of the metric space $X$ as a quotient $X=M / G$. In particular, if $X$ is a Riemannian orbifold then the quotient-geodesics are exactly the orbifold-geodesics of $X$.

On any $G$-horizontal geodesic $\gamma$, all but discretely many points have the same isotropy group $H=G_{\gamma}$. Hence the projection of $\gamma$ to $X$ is completely contained in the closure of the 
$H$-isotropy stratum of $X$. Therefore, the closure of any isotropy stratum is totally quotientgeodesic in $X$, in the sense that any quotient geodesic contained in the closure of the stratum initially, is contained in it for the whole time.

We would like to mention that the above observation about the dependence of the quotientgeodesics only on the metric structure of the quotient answers Question 4.6(1) in [AKLM03].

2.6. Luna-Richardson-Straume and minimal reductions. If $H$ is the principal isotropy group of the action of $G$ on $M$, then we have a canonical isometry $F / N \rightarrow M / G$, where $F$ is the closure of the set of all points of $M$ whose isotropy group is exactly $H$, and $N$ is the normalizer of $H$ in $G$ [LR79, Str94, GS00, GL14]. The action of $N$ on $F$ has $H$ in its kernel; the induced action of $N / H$ on $F$ is called the principal isotropy reduction of the action of $G$ on $M$. It has trivial principal isotropy groups and the same quotient space as the original action.

In case of a linear representation of a compact Lie group $G$ on a vector space $V$, the subset $F$ of $V$ is a subspace which we denote by $W$. Some properties of such and more general reductions (see below) have been investigated in GL14 to which we refer the reader for details. We will use the following special case of the results therein.

Lemma 2.2. Let $\rho: G \rightarrow \mathbf{O}(V)$ be a representation of a compact Lie group $G$. Assume $G$ is connected and its action on $V$ is irreducible, non-polar, infinitesimally polar and has cohomogeneity $c$ at least 4 . Let $H, N, W$ be as above. Then the action of the identity component $(\mathrm{N} / \mathrm{H})^{0}$ on $\mathrm{W}$ is irreducible.

Proof. Suppose, to the contrary, that the action of $(N / H)^{0}$ on $W$ is reducible. Since $G$ is connected and $V / G=W / N$, Theorem 1.7 in GL14 implies that $(N / H)^{0}$ is a torus $T^{c-2}$ and its action on $W$ is equivalent to the action of the maximal torus of $\mathbf{S U}(c-1)$ on $\mathbb{C}^{c-1}$. However, this action is not infinitesimally polar for $c \geq 4$ [GL14, Lemma 7.1], and this is a contradiction.

In a very last step of the proof of main theorems we will need to go through some lists of representations. To simplify the arguments excluding those candidates one can make use of the following slightly more general minimal reductions studied in GL14. For a representation $\rho: G \rightarrow \mathbf{O}(V)$, a minimal reduction of $\rho$ is a representation $\tau: K \rightarrow \mathbf{O}(U)$ with the same orbit space, $V / G=U / K$, and lowest possible dimension of $K$. It often happens that the principal isotropy reduction is already a minimal reduction, but there are easy examples in which it is not the case GL14].

Note that the representation $\rho$ is infinitesimally polar if and only if so is its minimal reduction $\tau$. Moreover, Lemma 2.2 applies to this situation as well, showing in particular that if $G$ is connected and its action on $V$ is irreducible and infinitesimally polar then its minimal reduction group $K$ cannot have a non-trivial toric connected component, unless the cohomogeneity of $\rho$ is at most 3 .

2.7. Infinitesimally polar representations with trivial principal isotropy groups. The principal isotropy/minimal reduction allows us to reduce some questions to the case of actions with trivial principal isotropy groups. In such a case, all slice representations must have trivial principal isotropy groups as well. On the other hand, if the quotient space is Riemannian orbifold then all isotropy representations are polar. But polar representations with trivial principal isotropy groups are exceedingly rare: 
Lemma 2.3. Let $\rho$ be an effective polar representation of a compact connected Lie group $G$ on $V$. Assume that the principal isotropy group of the representation is trivial. Then $G$ is finitely covered by $\mathbf{U}(1)^{l_{1}} \times \mathbf{S p}(1)^{l_{2}}$ and $\rho$ is orbit equivalent to $\left(l_{1} \cdot \mathbb{C} \oplus l_{2} \cdot \mathbb{H} \oplus l_{3} \cdot \mathbb{R}\right)$, where $\mathbb{R}$ denotes the trivial representation. Moreover there exists a connected normal subgroup $H$ of $G$ of rank 1 which is the isotropy group corresponding to a codimension 1-stratum of the quotient space $V / G=\left(\mathbb{R}^{+}\right)^{l_{1}+l_{2}} \times \mathbb{R}^{l_{3}}$. Finally, either $G$ is a torus, or $H$ can be chosen to be isomorphic to $\mathbf{S p}(1)$.

Proof. We prove the first assertion by induction on the number of irreducible components of $V$. Without loss of generality, we may assume that $V$ has no trivial components. Consider first the case in which $V$ is irreducible. A quick enumeration of polar irreducible representations of connected groups yields that $(G, V)$ is equal to $(\mathbf{U}(1), \mathbb{C})$ or $(\mathbf{S p}(1), \mathbb{H})$. In fact, we have only to examine the list of isotropy representations of compact irreducible symmetric spaces of maximal rank, which is a short list, and the list of additional polar irreducible representations in [EH99, which is even shorter.

Assume next $V=V_{1} \oplus V_{2}$ is a decomposition into $G$-invariant subspaces. Choose a regular point $v_{i} \in V_{i}$ for the restriction $\left(G, V_{i}\right)$ for $i=1,2$. Owing to [Dad85, Theorem 4], $\left(G_{v_{2}}^{0}, V_{1}\right)$ (resp. $\left.\left(G_{v_{1}}^{0}, V_{2}\right)\right)$ is orbit equivalent to $\left(G, V_{1}\right)$ (resp. $\left(G, V_{2}\right)$ ). The assumption of triviality of principal isotropy groups for $(G, V)$ implies that $\left(G_{v_{2}}^{0}, V_{1}\right)$ (resp. $\left(G_{v_{1}}^{0}, V_{2}\right)$ ) has the same property and it is effective; of course, it is also polar. It follows from the inductive hypothesis that $G_{v_{2}}^{0} \times G_{v_{1}}^{0}$ is covered by $\mathbf{U}(1)^{l_{1}} \times \mathbf{S p}(1)^{l_{2}}$ and its action on $V$ is orbit equivalent to $l_{1} \cdot \mathbb{C} \oplus l_{2} \cdot \mathbb{H}$. Again by [Dad85, Theorem 4], $G=G_{v_{2}}^{0} \cdot G_{v_{1}}^{0}=G_{v_{1}}^{0} \cdot G_{v_{2}}^{0}$ and $(G, V)$ is orbit equivalent to $\left(G_{v_{2}}^{0}, V_{1}\right) \times\left(G_{v_{1}}^{0}, V_{2}\right)$. Since $(G, V)$ has trivial principal isotropy groups, $G_{v_{1}}^{0} \cap G_{v_{2}}^{0}=\{1\}$, showing that $G$ is (finitely) covered by $G_{v_{1}}^{0} \times G_{v_{2}}^{0}$.

Note that the action of $\tilde{G}:=\mathbf{U}(1)^{l_{1}} \times \mathbf{S p}(1)^{l_{2}}$ on $V=l_{1} \cdot \mathbb{C} \oplus l_{2} \cdot \mathbb{H}$ needs not be a direct product of representations. However, $G$ acts on each $\mathbb{C}$-summand as $\mathbf{U}(1)$; in particular $\operatorname{Sp}(1)^{l_{2}}$ acts trivially on $l_{1} \cdot \mathbb{C}$. On the other hand, $G$ acts on each $\mathbb{H}$-summand of $V$ as one of $\mathbf{S p}(1), \mathbf{U}(2), \mathbf{S O}(4)$.

We next prove the second assertion in the statement. The claim is that we can find a $\mathbf{U}(1)-$ or $\mathbf{S p}(1)$-normal subgroup $H$ of $G$ which fixes all summands but one in the decomposition $V=l_{1} \cdot \mathbb{C} \oplus l_{2} \cdot \mathbb{H} \oplus l_{3} \cdot \mathbb{R}$. Again we may assume $l_{3}=0$. If $l_{2}=0$ then $\rho(G)$ is the maximal torus of $\mathbf{U}\left(l_{1}\right)$, the representation can be written as the $l_{1}$-fold direct product of the representation of $\mathbf{U}(1)$ on $\mathbb{C}$ and we can take $H$ to be any of these $\mathbf{U}(1)$-factors. Consider now $l_{2}>0$, let $G_{1}, \ldots, G_{l_{2}}$ denote the $\mathbf{S p}(1)$-factors of $G$ and let $V_{1}, \ldots, V_{l_{2}}$ denote the $\mathbb{H}$-summands of $V$. Each $G_{i}$ can act non-trivially only on the $\mathbb{H}$-summands. Thus assuming, contrarily to the claim, that no such factor corresponds to a codimension one stratum, we see that each $G_{i}$ acts on at least two $V_{j}$ 's. However, on each $V_{j}$ at most two of the $G_{i}$ 's can act non-trivially. Thus each $G_{i}$ acts non-trivially exactly on two $V_{j}$ 's. By relabeling, we may assume that there exists an integer $m$ such that $2 \leq m \leq l_{2}$ and $G_{i}$ acts non-trivially on $V_{i}$, $V_{i+1}$ for $i=1, \ldots, m-1$ and $G_{m}$ acts on $V_{m}, V_{1}$. Now the action of $G^{\prime}=G_{1} \times \cdots \times G_{m}$ on $V^{\prime}=V_{1} \oplus \cdots \oplus V_{m}$ is a direct factor of the action of $G$ on $V$. However, $\left(G^{\prime}, V^{\prime}\right)$ has principal isotropy subgroup $\mathbf{S O}(2)$, given by a maximal torus in the diagonal $\mathbf{S p}(1)$-subgroup of $G^{\prime}$, and this contradicts the fact that the original representation has trivial principal isotropy groups.

The next lemma will allow us to apply Lemma 2.3 to certain slice representations. 
Lemma 2.4. Let a connected Lie group $G$ act by isometries on a simply connected Riemannian manifold $M$. Assume that the principal isotropy groups of $(G, M)$ are trivial. If the quotient $M / G$ is a good Riemannian orbifold then all isotropy groups of $(G, M)$ are connected.

Proof. From [Lyt10, Theorem 1.8] we know that all slice representations have connected orbits. Since the slice representations have trivial principal isotropy groups, all isotropy groups must be connected.

\section{Discussion of the EXAMPles}

In this section we discuss the representations of compact connected groups $G$ appearing in Theorem 1.4.

3.1. Polar representations. They are listed in [Wol84, tables 8.11.2 and 8.11.5] and [EH99, Ber01, [FGT12], and their properties are well known by now. These are exactly the representations whose spherical quotient has constant curvature 1.

3.2. Almost free actions. We call a representation $\rho$ of the group $G$ almost free, if $G$ acts almost freely on the corresponding unit sphere. In this case $G$ has rank one (Lemma 2.1), hence it is either $\mathbf{U}(1)$, or it is covered by $\mathbf{S U}(2)$.

If $G=\mathbf{U}(1)$ then any almost free representation is the sum of non-trivial irreducible ones which are all complex one-dimensional. Such irreducible representations are naturally parametrized by positive integers $r$, the order of the kernel. The quotient space $X^{2 l}=$ $\mathbb{S}^{2 l+1} / \rho(\mathbf{U}(1))$ is called a weighted complex projective space, with weights $r_{1}, \ldots, r_{l+1}$, where $r_{1}, \ldots, r_{l+1}$ are the integers associated to each one of the summands of $\rho$.

If $G$ is covered by $\mathbf{S U}(2)$ then all almost free representations are described similarly (cf. [GG88]). It turns out that any almost free representation $\rho$ of $G$ is a sum of irreducible representations of quaternionic type and $G=\mathbf{S U}(2)$. For any natural number $r \geq 0$ there exists exactly one such irreducible representation of complex dimension $2 r$; the almost free representations of $\mathbf{S U}(2)$ are arbitrary sums of such representations. It seems natural to call the quotient space $X^{4 l}=\mathbb{S}^{4 l+3} / \rho(\mathbf{S U}(2))$ a weighted quaternionic projective space, also in view of the following remark.

Remark 3.1. The topology of the weighted projective spaces is very close to the topology of the usual projective spaces. In both cases the orbifold cohomology of the quotient $X$ can be computed using the fibration $G \rightarrow \mathbb{S}^{n} \rightarrow \hat{X}$, where $\hat{X}$ is Haefliger's classifying space of the orbifold $X$. The cohomology of $\hat{X}$, which by definition coincides with the orbifold cohomology of $X$, is generated by one element $e$ in degree 2 or 4 , respectively. The only relation is $p \cdot e^{k}=0$ for some natural number $p$. This number $p$ is 1 if and only if the representation acts freely on the sphere, i.e., if and only if the representation is a Hopf action. In any case $X$ has the same rational homotopy type as the corresponding projective space.

All weighted projective spaces have trivial orbifold fundamental group. Thus they are good orbifolds if and only if they are Riemannian manifolds. This happens if and only if the action of $G$ on $\mathbb{S}^{n}$ is free, i.e., if and only if the quotient $X$ is a classical projective space. Thus $X$ is diffeomorphic to a sphere if and only if $l=1$, in which case it has constant curvature 4 . 
3.3. Non-polar representations of cohomogeneity 3. This case is studied in detail in Str94. There it is shown that any such representation has a reduction to a representation of a one-dimensional group on $\mathbb{R}^{4}$. In fact, if $G \neq \mathbf{U}(1)$ then the reduction is either a finite extension of the Hopf action or a two-fold extension of the action of $\mathbf{U}(1)$ on $\mathbb{C} \oplus \mathbb{C}=\mathbb{R}^{4}$ with parameters $(1,2)$. In the second case, the quotient space $X$ is a disc with one singularity with angle $\pi / 2$. In any case, if $X$ is a good orbifold, then it has constant curvature 4 .

3.4. Complex case. We discuss here the second and the third examples from Table 1 (see also [Rad14, Prop. 5.2] where the same result is proven from a very different point of view). First we consider the double of the vector representation of $\mathbf{U}(2)$. We view $\mathbb{S}^{7} \subset \mathbb{H}^{2}$ and $\mathbf{U}(2)$ as left multiplication by a unit quaternion and right multiplication by a unit complex number. To obtain $\mathbb{S}^{7} / \mathbf{U}(2)$ we first divide out $\mathbf{S U}(2)$ to obtain $\mathbb{H} P^{1}$, the round sphere of curvature 4 and then we have to divide out the remaining isometric action of $\mathbf{U}(1)=\mathbf{U}(2) / \mathbf{S U}(2)$ on $\mathbb{H} P^{1}$.

In homogeneous coordinates $\left(q_{0}: q_{1}\right) e^{i \theta}=\left(q_{0} e^{i \theta}: q_{1} e^{i \theta}\right) \in \mathbb{H} P^{1}$ corresponds to $e^{-i \theta} q_{0}^{-1} q_{1} e^{i \theta} \in$ $\mathbb{H} \cup\{\infty\}=\mathbb{S}^{4}\left(\frac{1}{2}\right)$ and so $e^{i \theta} \in \mathbf{U}(1)$ fixes the plane spanned by $1, i$ and rotates the plane spanned by $j, k$ by $2 \theta$. Hence the action of $\mathbf{U}(1)$ on $\mathbb{H} P^{1}$ is polar and the quotient is a 3-dimensional hemisphere $\mathbb{S}_{+}^{3}\left(\frac{1}{2}\right)$ of constant curvature 4 .

The group $\mathbf{S U}(n)$ acting on $\mathbb{C}^{n} \oplus \mathbb{C}^{n}$ is orbit-equivalent to $\mathbf{U}(n)$ for $n \geq 3$. Moreover, we can apply the reduction procedure to $\mathbf{U}(n)$ and obtain $\left(\mathbf{U}(2), \mathbb{C}^{2} \oplus \mathbb{C}^{2}\right)$ as a minimal reduction. Hence the second and third representations in Table 1 yield a quotient isometric to $\mathbb{S}_{+}^{3}\left(\frac{1}{2}\right)$.

3.5. Quaternionic case. Herein we deal with the four last representations in Table 1; again a different approach can be found in [Rad14, Prop. 5.2].

Consider first $\mathbf{S p}(2)$ acting on $V=\mathbb{H}^{2} \oplus \mathbb{H}^{2}$. It is a reduction of $\left(\mathbf{S p}(n), \mathbb{H}^{n} \oplus \mathbb{H}^{n}\right)$ for $n \geq 3$, so the quotient $X=\mathbb{S}^{8 n-1} / \mathbf{S p}(n)=\mathbb{S}^{15} / \mathbf{S p}(2)$ has a non-empty boundary. The representation has trivial principal isotropy group, hence $X$ has dimension 5 . The normalizer of $\mathbf{S p}(2)$ in $\mathbf{O}(V)$ equals $\mathbf{S p}(2) \cdot \mathbf{S p}(2)$. Thus we have a non-trivial action of $\mathbf{S p}(2)$ on $X$ which leaves the boundary invariant, fixes the unique point $x$ of maximal distance to $\partial X$, and acts as $\mathbf{S p}(2) / \mathbb{Z}_{2}=\mathbf{S O}(5)$ on the tangent space at $x$. The quotient of the $\mathbf{S p}(2)$-action on $X$ is given by $\mathbb{S}^{15} / \mathbf{S p}(2) \cdot \mathbf{S p}(2)$. This space is known to be an interval of length $\left[0, \frac{\pi}{4}\right]$, which says that $\mathbf{S O}(5)$ acts on $X$ with cohomogeneity one. Now we see that $X$ must be a hemi-sphere with a rotationally invariant metric; we are going to show that it has constant curvature 4.

Let $\gamma:\left[0, \frac{\pi}{4}\right] \rightarrow V$ be the horizontal geodesic

$$
\gamma(r)=\left(\begin{array}{cc}
\cos r & 0 \\
0 & \sin r
\end{array}\right)
$$

Its projection under $\pi: \mathbb{S}^{15} \rightarrow X$ starts at a boundary point of $X$ and ends at $x$. Note that $\pi^{-1}(\partial X)$ consists of pairs of linearly dependent vectors over $\mathbb{H}, \pi\left(\left\{\left(\begin{array}{ll}* & * \\ 0 & 0\end{array}\right)\right\}\right)=\partial X$ and

$$
\partial X=\left\{\left(\begin{array}{ll}
* & * \\
0 & 0
\end{array}\right)\right\} /\left(\begin{array}{cc}
\mathbf{S p}(1) & 0 \\
0 & 1
\end{array}\right)=\mathbb{S}^{7} / \mathbf{S p}(1)=\mathbb{H} P^{1}=\mathbb{S}^{4}(1 / 2) .
$$

The $\mathbf{S O}(5)$-orbit through $\pi(\gamma(r))$ for $0<r<\frac{\pi}{4}$ is a 4 -sphere of constant curvature. In fact, due to the presence of $\mathbf{S O}(5)$-symmetry the metric on $X$ has the form $d r^{2}+f^{2}(r) \omega$, where 
$\omega$ is the metric of constant curvature 4 on $\mathbb{S}^{4}$ and $f$ is a non-negative function. Moreover, $f(r)$ is equal to the quotient $\left\|\pi_{*} \xi\right\|_{\pi(\gamma(r))} /\left\|\pi_{*} \xi\right\|_{\pi(\gamma(0))}$, for an arbitrary Killing field $\xi$ on $\mathbb{S}^{15}$ induced by the Lie algebra $\mathfrak{s p}(2)$ acting on the right whose $\pi$-horizontal component does not vanish at $\gamma(0)$. Now we consider the unit Killing field $\xi$ induced by

$$
\left(\begin{array}{cc}
0 & 1 \\
-1 & 0
\end{array}\right)
$$

(quaternionic matrix) multiplying $\gamma(r)$ on the right. This Killing field is not $\pi$-horizontal with respect to $\pi$. Its $\pi$-vertical component is parallel to the unit Killing field $\eta$ on $\mathbb{S}^{15}$ induced by the same matrix multiplying $\gamma(r)$ on the left. Taking scalar products, the $\pi$ vertical component of $\xi$ has length $\sin (2 r)$ and the $\pi$-horizontal one, $\cos (2 r)$. It follows that $f(r)=\cos (2 r)$. Hence we see that $X$ is a hemi-sphere $\mathbb{S}_{+}^{5}(1 / 2)$ of constant curvature 4 .

Similarly, the last three representations in Table 1 have reductions to the respective representations with $n=2$, so we may assume $n=2$ in the discussion of the remaining orbit spaces. In turn, these orbit spaces are obtained by dividing $X$ by a subgroup of $\mathbf{S p}(2)$ acting on $X$ whose action is induced by right multiplication on $V$. Namely, we consider $\mathbf{S p}(1) \times \mathbf{S p}(1), \Delta_{\mathbf{S p}(1)}$ (diagonal), $\mathbf{T}^{2}$, and $\Delta_{\mathbf{T}^{1}}=\mathbf{U}(1)$; here the first group is included only for the sake of completeness, whereas the remaining three yield the representations of interest to us. These groups fix $x$, so it is enough to understand the action on $T_{x} X$ : this is given by conjugation. Note that $T_{x} X \cong \mathcal{H}_{x}=\left\langle e_{0}, e_{1}, e_{2}, e_{3}, e_{4}\right\rangle$ where

$$
e_{0}=\left(\begin{array}{cc}
1 & 0 \\
0 & -1
\end{array}\right), e_{1}=\left(\begin{array}{ll}
0 & 1 \\
1 & 0
\end{array}\right), e_{2}=\left(\begin{array}{cc}
0 & -i \\
i & 0
\end{array}\right), e_{3}=\left(\begin{array}{cc}
0 & -j \\
j & 0
\end{array}\right), e_{4}=\left(\begin{array}{cc}
0 & -k \\
k & 0
\end{array}\right) \text {. }
$$

Let $a, b \in \mathbb{H}$. Then:

$$
\mathbf{U}(1):\left(\begin{array}{cc}
e^{-i \theta} & 0 \\
0 & e^{-i \theta}
\end{array}\right)\left(\begin{array}{ll}
0 & a \\
b & 0
\end{array}\right)\left(\begin{array}{cc}
e^{i \theta} & 0 \\
0 & e^{i \theta}
\end{array}\right)=\left(\begin{array}{cc}
0 & e^{-i \theta} a e^{i \theta} \\
e^{-i \theta} b e^{i \theta} & 0
\end{array}\right)
$$

fixes $\left\langle e_{0}, e_{1}, e_{2}\right\rangle$ and acts as $\mathbf{S O}(2)$ on $\left\langle e_{3}, e_{4}\right\rangle$;

$$
\mathbf{T}^{2}:\left(\begin{array}{cc}
e^{-i \theta} & 0 \\
0 & e^{-i \varphi}
\end{array}\right)\left(\begin{array}{ll}
0 & a \\
b & 0
\end{array}\right)\left(\begin{array}{cc}
e^{i \theta} & 0 \\
0 & e^{i \varphi}
\end{array}\right)=\left(\begin{array}{cc}
0 & e^{-i \theta} a e^{i \varphi} \\
e^{-i \varphi} b e^{i \theta} & 0
\end{array}\right)
$$

fixes $\left\langle e_{0}\right\rangle$ and acts as $\mathbf{S O}(2) \times \mathbf{S O}(2)$ on $\left\langle e_{1}, e_{2}\right\rangle \oplus\left\langle e_{3}, e_{4}\right\rangle$;

$$
\mathbf{S p}(1):\left(\begin{array}{cc}
q^{-1} & 0 \\
0 & q^{-1}
\end{array}\right)\left(\begin{array}{ll}
0 & a \\
b & 0
\end{array}\right)\left(\begin{array}{ll}
q & 0 \\
0 & q
\end{array}\right)=\left(\begin{array}{cc}
0 & q^{-1} a q \\
q^{-1} b q & 0
\end{array}\right)
$$

fixes $\left\langle e_{0}, e_{1}\right\rangle$ and acts as $\mathbf{S O}(3)$ on $\left\langle e_{2}, e_{3}, e_{4}\right\rangle$;

$$
\mathbf{S p}(1) \times \mathbf{S p}(1):\left(\begin{array}{cc}
q_{1}^{-1} & 0 \\
0 & q_{2}^{-1}
\end{array}\right)\left(\begin{array}{cc}
0 & a \\
b & 0
\end{array}\right)\left(\begin{array}{cc}
q_{1} & 0 \\
0 & q_{2}
\end{array}\right)=\left(\begin{array}{cc}
0 & q_{1}^{-1} a q_{2} \\
q_{2}^{-1} b q_{1} & 0
\end{array}\right)
$$

fixes $\left\langle e_{0}\right\rangle$ and acts as $\mathbf{S O}(4)$ on $\left\langle e_{1}, e_{2}, e_{3}, e_{4}\right\rangle$.

It follows that the quotient of $X$ by those groups is respectively

$$
\mathbb{S}_{++}^{4}(1 / 2), \mathbb{S}_{+++}^{3}(1 / 2), \mathbb{S}_{++}^{3}(1 / 2), \mathbb{S}_{++}^{2}(1 / 2)
$$

(cf. [Str94, Table II, type II] in the last case). 
3.6. Exceptional case. Finally, we deal with the action of $G=\operatorname{Spin}(9)$ on $V=\mathbb{R}^{16} \oplus \mathbb{R}^{16}$.

Let $H$ be the principal isotropy group, $W=V^{H}$ its fixed point set. The isotropy subgroup at a point in $\mathbb{R}^{16} \oplus\{0\}$ is $\operatorname{Spin}(7)$. The action of $\operatorname{Spin}(7)$ on $\{0\} \oplus \mathbb{R}^{16}$ decomposes as $\mathbb{R} \oplus \mathbb{R}^{7} \oplus \mathbb{R}^{8}$, the sum of the vector and spin representations plus a trivial component. This is a slice representation which is polar, and corresponds to a unique non-principal minimal orbit type. It follows that our representation is infinitesimally polar. It also follows that $H \cong \mathbf{S U}(3)$ and thus the dimension of $X=\mathbb{S}^{31} / G$ is 3 .

The identity component of the normalizer $N_{G}(H)^{0}=H \cdot Z_{G}(H)^{0}$, where $Z_{G}(H)$ denotes the centralizer of $H$ in $G$. One computes that $Z_{G}(H)^{0} \cong \mathbf{U}(2)$ (for instance, by using root systems; alternatively compare Gor08, p. 137]). Let $C:=\left(N_{G}(H) / H\right)^{0}$. Then $C \cong \mathbf{U}(2)$ and $\operatorname{dim} W=8$. Since $(G, V)$ is a doubling representation, so is $(C, W)$; hence $W=\mathbb{C}^{2} \oplus \mathbb{C}^{2}$ (one can also refer to Proposition 6.1 below or [Gor08]).

Now $X^{\prime}=W / C=\mathbb{S}_{+}^{3}\left(\frac{1}{2}\right)$ due to subsection 3.4 , and $X=\mathbb{S}^{31} / G$ is a finite quotient of $X^{\prime}=W / C$ by a group $\Gamma$ generated by reflections [GL14, Prop. 1.2]. Note that $\Gamma$ is non-trivial as $(G, V)$ has more than two orbit types.

There is an isometric $\mathbf{S O}(2)$ action on $X$ with quotient $X / \mathbf{S O}(2)=\mathbb{S}^{2}\left(\frac{1}{2}\right)_{+++}$. Indeed there is a circle in the centralizer of $\mathbf{S p i n}(9)$ in $\mathbf{O}(V)$, and $\mathbb{S}^{31} / \mathbf{S p i n}(9) \times \mathbf{S O}(2)$ is a spherical triangle with three right angles [Str94, Table II, type $\mathrm{III}_{4}$ ].

The isometry group of $\mathbb{S}^{3}\left(\frac{1}{2}\right)_{+}$is $\mathbf{O}(3)$ acting through the canonical action on the twodimensional boundary sphere. The reflections of $X$ are exactly the reflections in $\mathbf{O}(3)$. If $\Gamma$ has more than two elements, it contains two different reflections, and the quotient of the boundary sphere by $\Gamma$ does not admit a circle action, hence this case cannot occur. Hence $\Gamma$ has two elements, the identity and a reflection. All reflections in the isometry group of $\mathbb{S}^{3}\left(\frac{1}{2}\right)_{+}$are conjugate and the quotient of $X^{\prime}$ under the action of any such reflection is thus $X=\mathbb{S}^{3}\left(\frac{1}{2}\right)_{++}$.

Remark 3.2. A conceptual proof that the above orbit spaces have constant curvature (specially in the case of simple groups, see [Gor08]) is to check that the representations are taut and infinitesimally polar, and use [Wie14, Theorem 3.20] and [TT97, Corollary 6.12].

\section{Positively CURVEd ORBIFolds}

4.1. Coxeter orbifolds. All orbifolds below are considered with some complete Riemannian metric. Recall that an orbifold is called a Coxeter orbifold if all of its local groups are finite Coxeter groups acting as reflection groups on the corresponding tangent spaces. In [Dav10] such orbifolds are called reflectofolds. We denote by $X_{\text {reg }}$ the set of manifold points of an orbifold.

We start with some preliminaries:

Lemma 4.1. An orbifold $X$ is a Coxeter orbifold if and only if $X \backslash X_{\text {reg }}=\partial X$.

Proof. The claim and conditions are local. Thus we may assume that $X$ has the form $X=\mathbb{R}^{n} / \Gamma$ for a finite group $\Gamma$ of linear isometries.

The only if direction is clear, due to the structure of Coxeter chambers. Assume now that any non-regular point of $X$ is contained in $\partial X$. Let $\Gamma_{r e f l}$ be the normal subgroup of $\Gamma$ generated by reflections. Thus $\Gamma_{r e f l}$ is a finite reflection group and we need to prove $\Gamma=\Gamma_{\text {refl }}$.

Consider the Coxeter chamber $X^{\prime}=\mathbb{R}^{n} / \Gamma_{\text {refl }}$ and the induced effective, isometric action of $\Gamma^{\prime}=\Gamma / \Gamma_{\text {refl }}$ on the orbifold $X^{\prime}$. The set of regular points $X_{\text {reg }}^{\prime}$ is homeomorphic to $\mathbb{R}^{n}$, and 
it is preserved under the action of $\Gamma^{\prime}$. Hence, if $\Gamma^{\prime}$ is non-trivial, the action of the finite group $\Gamma^{\prime}$ on the contractible manifold $X_{r e g}^{\prime}$ cannot be free. On the other hand, $\Gamma^{\prime}$ has no reflections, thus the set of fixed points in $X_{r e g}^{\prime}$ of any non-trivial element in $\Gamma^{\prime}$ has codimension at least two. Therefore, the image of this set of fixed points in $X$ consists of singular points outside of $\partial X$.

We deduce that this set of fixed points is empty, hence the action of $\Gamma^{\prime}$ on $X_{r e g}^{\prime}$ is free, hence $\Gamma^{\prime}$ is the trivial group and $\Gamma=\Gamma_{\text {refl }}$.

The above lemma is closely related to the following result which we quote from Lyt10, Theorem 1.8]:

Lemma 4.2. Let the connected group $G$ act by isometries on a simply connected Riemannian manifold $M$. Assume that the quotient $X=M / G$ is a Riemannian orbifold. The orbifold $X$ is a Coxeter orbifold if and only if $X \backslash \partial X$ is a good orbifold. In particular, if $X$ is a good orbifold, it is automatically a Coxeter orbifold.

For an orbifold $X$ consider the universal covering $\hat{X}$ of the underlying topological space with the induced orbifold structure. Since being a Coxeter orbifold is a local condition, $X$ is a Coxeter orbifold if and only if $\hat{X}$ is a Coxeter orbifold. The universal orbi-covering $\tilde{X}$ is also the universal orbi-covering of $\hat{X}$. Hence investigating the question, whether a given Coxeter orbifold $X$ is a good orbifold we may restrict to the case, when the underlying topological space $X$ is simply connected.

For a Coxeter orbifold $X$, the faces are by definition the strata of codimension 1 . The closure of a face is called a mirror. Then each singular point of $X$ is contained in some mirror. Given any point $x \in X$, consider a small open convex ball $U$ around $x$. Since $U$ is diffeomorphic as an orbifold to a Coxeter chamber, we see that $x$ is contained in exactly $k$ mirrors of the orbifold $U$ if and only if $x$ lies on a stratum of codimension $k$. Note that a face of $U$ is part of a face of $X$, but that different faces of $U$ could be part of the same face of $X$.

It is easy to characterize which Coxeter orbifolds with simply connected underlying space are good, expressed in terms of the intersections of mirrors. One part of the following result is straightforward ([Dav10], Section 5.1); the other one is contained in [AKLM07], Corollary 6.5. (In AKLM07, the result is unfortunately incorrectly stated without the assumptions (C1), (C2) below. However, the assumptions are implicitly made in Section 4 of [AKLM07], where they are wrongly assumed to hold always.) For convenience of the reader, we recall the proof of this result:

Lemma 4.3. Let $X$ be a Coxeter orbifold with $\pi_{1}(X)=1$. The orbifold $X$ is good if and only if it satisfies the following conditions:

(C1) Any codimension two stratum is contained in two different mirrors.

(C2) If the intersection $\bar{W} \cap \bar{W}^{\prime}$ of two different mirrors contains two different strata $B^{ \pm}$ of codimension 2 , then the orders of the isotropy groups at $B^{+}$and at $B^{-}$coincide.

Proof. The orbifold fundamental group $\Gamma$ of $X$ is generated by involutions $s_{W}$, indexed by the faces $W$ of $X$ (cf. [Dav10]). The relations are generated by words of the form $r_{S}$, indexed by strata $S$ of codimension 2. For any such stratum $S$, consider a point $x \in S$ and a small neighborhood $U$ of $x$. Then the word $r_{S}$ has the form $r_{S}=\left(s_{W} \cdot s_{W^{\prime}}\right)^{n}$, where $n$ is the dihedral angle at $x$ (half of the order of the local isotropy group $\Gamma_{x}$ ) and where $W$ and $W^{\prime}$ are the faces that extend the local faces in $U$ adjacent to $x$. 
For any $x \in X$ the local group $\Gamma_{x}$ is the finite Coxeter group generated by the involutions indexed by the local faces at $x$. And the canonical map $\Gamma_{x} \rightarrow \Gamma$ sends the generator of $\Gamma_{x}$ corresponding to a local face to the generator of $\Gamma$ corresponding to the global face which extends the local one.

The orbifold $X$ is good if and only if all maps $\Gamma_{x} \rightarrow \Gamma$ are injective. If one of conditions $(\mathrm{C} 1)$ or (C2) does not hold, then the map from $\Gamma_{x}$ to $\Gamma$ is not injective, where $x$ is any point in a codimension 2 stratum appearing in (C1), respectively, in the stratum among $B^{+}, B^{-}$at which the local isotropy group is larger in case $(\mathrm{C} 2)$.

On the other hand, if $(\mathrm{C} 1)$ and $(\mathrm{C} 2)$ hold true then $\Gamma$ is the Coxeter group with the Coxeter generating system $\left\langle s_{W} \mid r_{S}\right\rangle$, and any local group $\Gamma_{x}$ is given by a Coxeter subsystem. The statement that $\Gamma_{x} \rightarrow \Gamma$ is injective is a standard statement about Coxeter groups.

Let again $X$ be a Coxeter orbifold with $\pi_{1}(X)=1$. Let $W$ be a face. Consider $W$ with its intrinsic metric and let $N$ be the completion. Looking at the situation locally, we see that $N$ is an Alexandrov space, which is in addition a Riemannian manifold with corners (cf. AKLM07). We have a canonical map $i_{W}: N \rightarrow X$, which is an isometric embedding in a neighborhood of any point of $N$. The image of $i_{W}$ is the mirror $\bar{W}$. The map $i_{W}$ is injective on $W$, but, a priori, it could happen, that the map $i_{W}$ is not injective on $N$.

The space $N$ considered as a manifold with corners has a canonical stratification, and any stratum of codimension $k$ is mapped by $i_{W}$ to a stratum of codimension $k+1$ in $X$. The closure of any stratum of $N$ is an extremal subset of $N$ in the sense of Alexandrov geometry. We consider the following condition on the orbifold $X$ :

(C3) For any face $W$, any pair of closures of codimension 1 strata in the completion $N$ of $W$ have a non-empty intersection in $N$.

Lemma 4.4. Let $X$ be a Coxeter orbifold. The condition (C3) implies (C1) and (C2).

Proof. Assume that (C1) does not hold. Consider a bad stratum $A$ of codimension 2, an adjacent face $W$ and the completion $N$. Then we have two different strata $A^{ \pm}$of codimension 1 in $N$ that are mapped by $i_{W}$ to the same stratum $A$. By assumption $\bar{A}^{+} \cap \bar{A}^{-}$is not empty. At any point $z$ in this intersection, the restriction of $i_{W}$ to any neighborhood of $z$ is noninjective, which is impossible.

Assume that (C2) does not hold and consider $W, W^{\prime}, B^{ \pm}$as in the definition. We claim that $\bar{B}^{+} \cap \bar{B}^{-}$is empty. Otherwise, in the neighborhood of any point $x$ in the intersection, we would see two local mirrors which intersect in different local strata of codimension 2. But this does not happen in a Coxeter chamber.

On the other hand, consider the completion $N$ of $W$ and the two strata $A^{ \pm}$in $N$ that are mapped by $i_{W}$ to $B^{ \pm}$respectively. Choose a point $z$ in the non-empty intersection $\bar{A}^{+} \cap \bar{A}^{-}$, which must exist by assumption (C3). Then the point $x=i_{W}(z)$ is contained in $\bar{B}^{+} \cap \bar{B}^{-}$, in contradiction to the previous argument.

4.2. Positively curved Coxeter orbifolds. Assume now that a compact Coxeter orbifold $X$ is positively curved. The completion $N$ of any face $W$ is positively curved as well. From the theorem of Petrunin-Frankel ([Pet98], Corollary 3.3) we deduce that any pair of strata of codimension 1 in $N$ has a non-empty intersection, if the dimension of $N$ is at least 2 and the curvature greater than some positive constant. Thus $X$ satisfies the condition (C3), if $\operatorname{dim}(X) \geq 3$. We can also apply this reasoning to the universal covering $\hat{X}$ of $X$ (since it has curvature bounded below by some positive constant, so it is a compact Coxeter orbifold as well). We arrive at: 
Lemma 4.5. Let $X$ be a compact positively curved Coxeter orbifold of dimension at least 3. Then $X$ is a good orbifold.

In the course of the proof of Theorem 1.5, we will be needing the following result whose proof is contained in the first few lines of the proof of Proposition 2.3 in [FGT12]:

Lemma 4.6. Let $M$ be a simply connected compact positively curved manifold. Let $g: M \rightarrow$ $M$ be a reflection at a totally geodesic hypersurface $N$. Then $M$ and $N$ are diffeomorphic to spheres.

Now we can prove Theorem 1.5 .

Proof of Theorem 1.5. Let $\tilde{X}$ denote the universal orbi-covering of $X$. By assumption, $\tilde{X}$ has uniformly positive curvature, in particular it is compact. There is at least one reflection $g$ on $\tilde{X}$. We may assume that $g$ is the reflection at a totally geodesic hypersurface $Z$, dividing $\tilde{X}$ in two isometric connected components. Without loss of generality, we may assume $X=\tilde{X} /\{1, g\}$. Then $X$ has boundary $\partial X=Z$ (as an Alexandrov space) and the orbifold $X_{0}:=X \backslash \partial X$ has trivial orbifold fundamental group (for instance, cf. [GL14, Prop. 3.4]).

We claim first that $X_{0}$ is a manifold. We refer to [KL11, pp.5-23] for details of the following argument. By positivity of curvature, the distance function $f=d_{Z}$ is strictly concave on $X_{0}$. It has a unique critical point $p$ (in the sense of Alexandrov geometry, which is equivalent to being critical in the orbifold sense). Moreover, $X_{0} \backslash\{p\}$ is diffeomorphic (as an orbifold) to the product $(0, t) \times T$, where $T$ is a level set of $f$. This implies that $\pi_{1}^{\text {orb }}\left(X_{0}\right)=\pi_{1}^{\text {orb }}\left(X_{0} \backslash\{p\}\right)=\pi_{1}^{\text {orb }}\left(U_{p}\right)$, for any small neighborhood $U_{p}$ of $p$. Since $\pi_{1}^{\text {orb }}\left(X_{0}\right)=1$, this shows that $p$ is a regular point of the orbifold $X_{0}$. Since the whole orbifold $X_{0}$ is diffeomorphic to an open suborbifold of $U_{p}$, we deduce that $X_{0}$ is a manifold.

Due to Lemma 4.1, $X$ a Coxeter orbifold. Due to Lemma 4.5 the orbifold $X$ is a good orbifold. Hence, $\tilde{X}$ is a manifold.

Now, $\tilde{X}$ is a positively curved manifold with an isometric reflection at a hypersurface. By Lemma 4.6 we deduce that $\tilde{X}$ must be diffeomorphic to a sphere.

\section{ORBIFOLDS ISOMETRIC TO QUOTIENTS OF SPHERES}

5.1. Reductions and immersions of strata. We consider a more general construction than that in subsection 2.6. Let a compact Lie group $G$ act by isometries on a complete connected Riemannian manifold $M$. Denote the quotient space $M / G$ by $X$. Let $H \subset G$ be the isotropy group of an arbitrary point in $M$. Denote the normalizer of $H$ in $G$ by $N$. Consider the set $F^{\prime}$ of fixed points of $H$, and let $F^{0} \subset F^{\prime}$ be the set of all points whose isotropy group is exactly $H$. Then $F^{\prime}$ is totally geodesic in $M, F^{0}$ is open in $F^{\prime}$ and the closure $F$ of $F^{0}$ is a union of some components of $F^{\prime}$. The sets $F$ and $F^{0}$ are $N$-invariant. The embedding $F \rightarrow M$ defines a canonical map $I_{H}: F / N \rightarrow M / G$. For a general, non-principal isotropy group $H$, the map $I$ needs not be injective, as the following typical example shows (the version of this map in the category of algebraic actions of complex reductive groups on affine varieties is a normalization map, cf. [Lun75, §2]).

Example 5.1. Consider the representation of $G=\mathbf{U}(2)$ on $\mathbb{C}^{2} \oplus \mathbb{R}^{3}$, where $G$ acts on $\mathbb{R}^{3}$ as $\mathbf{S O}(3)$ (Case 12 in Table 3 below; see also [Str94, Table II, type I]). Let $M$ be the unit sphere with the induced action of $G$. The quotient space $X=M / G$ is half of a tear-drop. It is topologically a disc, which has three isotropy strata, all of them connected: the open disc, the complement of one point on the boundary circle and this one point. The 1-dimensional 
stratum corresponds to the isotropy group $H=\mathbf{U}(1)$. The corresponding normalizer $N$ is the maximal torus $\mathbf{U}(1) \times \mathbf{U}(1)$. The set $F$ of fixed points is the unit sphere $\mathbb{S}^{2}$ in $\mathbb{C} \times \mathbb{R}$ and the quotient space $F / N$ is the interval of length $\pi$. The map $I$ sends this interval lengthpreserving onto the boundary of the disc and folds both end-points to the most singular point of $X$.

In general we have:

Lemma 5.1. Under the assumptions above, the map $I_{H}: F / N \rightarrow M / G$ is 1-Lipschitz, finiteto-one and preserves the lengths of curves. The restriction of $I_{H}$ to $F^{0} / N$ is an injective local isometry onto the $H$-isotropy stratum of $M / G$. Moreover, $I_{H}$ preserves the quotient-geodesic flow, i.e., it sends a projection of a horizontal geodesic to the projection of a horizontal geodesic.

Proof. The image $I_{H}\left(F^{0} / N\right)$ is the set of all $G$-orbits that contain a point with isotropy group $H$, hence exactly the $H$-isotropy stratum of $M / G$. If two points $p_{1}, p_{2} \in F_{0}$ are in the same $G$-orbit then any element $g \in G$ sending $p_{1}$ to $p_{2}$ must normalize $H$. Hence, $p_{1}$ and $p_{2}$ are in the same $N$-orbit. Thus the map $I_{H}$ is injective on $F_{0} / N$.

The remaining claims follow directly, once we know that $I_{H}$ preserves the quotient-geodesic flow. Since $F_{0} / N$ is dense in $F / N$ it suffices to see that an $N$-horizontal geodesic in $F^{0}$ is a $G$-horizontal geodesic in $M$. But this becomes clear by looking at the slice representation (of $H$ ) at any point of $F^{0}$.

5.2. The orbifold case. In case that the quotient space is an orbifold we deduce:

Corollary 5.2. Let a compact Lie group G act by isometries on a complete Riemannian manifold $M$. Assume that $X=M / G$ is a Riemannian orbifold. Let $H$ be any isotropy group of the action, with normalizer $N$ and $F$ defined as above. Then $F / N$ is a Riemannian orbifold. The map $I_{H}: F / N \rightarrow M / G$ is a totally geodesic isometric immersion of orbifolds.

Proof. The subset $F^{0} / N$ is open and dense in $F / N$. On the other hand, $I_{H}$ is a Riemannian isometry from $F^{0} / N$ onto its image. This image is a stratum in a Riemannian orbifold. We deduce that the sectional curvatures in the principal part of $F^{0} / N$ are uniformly bounded from both sides. Hence $F / N$ is a Riemannian orbifold by [LT10].

The second claim follows from the first claim, Lemma 5.1 and the fact that the quotientgeodesic flows in $X$ and in $F / N$ coincide with the respective orbifold-geodesic flows (Subsection 2.5).

5.3. Spherical case. Now we state the application of the strata immersion map $I_{H}$ which will be used in the inductive proof of our main step:

Corollary 5.3. Let the compact Lie group $G$ act isometrically on the round sphere $\mathbb{S}^{n}$. Assume that the action is non-polar and that the quotient $X=\mathbb{S}^{n} / G$ is a Riemannian orbifold of dimension $k \geq 3$ with a non-empty boundary. Then there exists a non-polar isometric action of a compact Lie group $N$ on some unit sphere $\mathbb{S}^{m}$, such that the quotient $Y=\mathbb{S}^{m} / N$ is a good Riemannian orbifold of dimension $k-1$. Moreover, there is a isometric reflection $r$ on the universal orbi-covering $\tilde{X}$ of $X$, whose set $Z$ of fixed points is the universal orbi-covering of $Y$. In particular, the universal orbi-covering of $Y$ is diffeomorphic to a sphere. 
Proof. Recall that $X$ is a good Riemannian orbifold and $\tilde{X}$ is diffeomorphic to a sphere by Theorem 1.5 .

Choose a point $p \in \mathbb{S}^{n}$ which is projected to a codimension one stratum $S t$ in $X$. Let $H$ be the isotropy group of $p$. Then the set of fixed points $F$ of $H$ is a great subsphere of $\mathbb{S}^{n}$ of some dimension $m$. The results above show that $Y=\mathbb{S}^{m} / N$ is Riemannian orbifold and that $I_{H}: Y \rightarrow X$ is an isometric (totally geodesic) immersion whose image contains $S t$. In particular, $Y$ has dimension $k-1$.

The map $I_{H}$ lifts to an isometric, totally geodesic immersion $\tilde{I}_{H}: Z \rightarrow \tilde{X}$, between the universal orbi-coverings of $Y$ and $X$, respectively [BH99, p. 611]. Since $\tilde{X}$ is a manifold, we deduce that $Z$ is a manifold. In particular, $Y$ is a good Riemannian orbifold.

Let $x \in \tilde{X}$ be any point projected to $S t$ in $x$. Then there is a isometric reflection $r: \tilde{X} \rightarrow \tilde{X}$ (belonging to $\pi_{1}^{\text {orb }}(X)$ ), whose set $Z^{\prime}$ of fixed points is a $(k-1)$-dimensional totally geodesic submanifold containing $x$, whose projection to $X$ contains $S t$. We see by dimensional reasons and the fact that $\tilde{I}_{H}$ is totally geodesic, that the image $\tilde{I}_{H}(Z)$ coincides with $Z^{\prime}$. The map $\tilde{I}_{H}: Z \rightarrow Z^{\prime}$ is a local isometry, hence a covering map. By Lemma 4.6, $Z^{\prime}$ is diffeomorphic to a sphere. Since $k \geq 3$, the sphere $Z^{\prime}$ is simply connected and $\tilde{I}_{H}$ is an isometry.

It only remains to prove that the action of $N$ on $\mathbb{S}^{m}$ is non-polar. Otherwise, $Y$ and hence $Z^{\prime}$ had constant curvature 1 . The submanifold $Z^{\prime}$ divides the manifold $\tilde{X}$ into two submanifolds with totally geodesic boundary $Z^{\prime}$. Since the curvature of $\tilde{X}$ is at least 1 , we can apply a special case of a theorem by Hang and Wang [HW09, Theorem 2] to see that $\tilde{X}$ has constant curvature 1 (cf. also [Pet07, (3.3.5)] for a closely related result). But then the action of $G$ on $\mathbb{S}^{n}$ is polar. This contradicts our assumptions.

\section{Special ReDucible CASE}

In this section we are going to analyze sums of representations of cohomogeneity one. Herein it is convenient to adopt a different notation for representations (compare [Str94]). We are going to prove:

Proposition 6.1. Let $\rho: G \rightarrow \mathbf{O}(V)$ be a representation of a compact connected Lie group $G$. Assume $V=V_{1} \oplus V_{2}$ is a $G$-irreducible decomposition such that the restrictions $\rho_{i}$ to $V_{i}$ have cohomogeneity 1. If $\rho$ is infinitesimally polar but not polar then $\rho$ is listed in the table below (orbit-equivalent representations are designated by the same number and different letters). In particular, the cohomogeneity of $\rho$ in $V$ is at most 6. 


\begin{tabular}{|c|c|c|c|c|}
\hline Case & $G$ & $\rho$ & Cohom & Conditions \\
\hline $1 a$ & $\mathbf{S O}(n)$ & $\rho_{n} \oplus \rho_{n}$ & 3 & $n \geq 2$ \\
$1 b$ & $\mathbf{G}_{2}$ & $\mathbb{R}^{7} \oplus \mathbb{R}^{7}$ & 3 & - \\
$1 c$ & $\mathbf{S p i n}(7)$ & $\Delta_{7} \oplus \Delta_{7}$ & 3 & - \\
2 & $\mathbf{S p i n}(9)$ & $\Delta_{9} \oplus \Delta_{9}$ & 4 & - \\
3 & $\mathbf{S U}(2)$ & $\mu_{2} \oplus \mu_{2}$ & 5 & - \\
$4 a$ & $\mathbf{S U}(n)$ & $\mu_{n} \oplus \mu_{n}$ & 4 & $n \geq 3$ \\
$4 b$ & $\mathbf{U}(n)$ & $\mu_{1} \otimes \mu_{n} \oplus \mu_{1} \otimes \mu_{n}$ & 4 & $n \geq 2$ \\
$5 a$ & $\mathbf{U}(1) \times \mathbf{S U}(n) \times \mathbf{U}(1)$ & $\mu_{1} \otimes \mu_{n} \oplus \mu_{n} \otimes \mu_{1}$ & 3 & $n \geq 2$ \\
$5 b$ & $\mathbf{U}(1) \times \mathbf{S U}(n)$ & $\left(\mu_{1}\right)^{r} \otimes \mu_{n} \oplus\left(\mu_{1}\right)^{s} \otimes \mu_{n}$ & 3 & $n \geq 3, r \neq s$ \\
6 & $\mathbf{S p}(n)$ & $\nu_{n} \oplus \nu_{n}$ & 6 & $n \geq 2$ \\
7 & $\mathbf{S p}(n) \times \mathbf{U}(1)$ & $\nu_{n} \otimes \mu_{1} \oplus \nu_{n} \otimes \mu_{1}$ & 5 & $n \geq 2$ \\
8 & $\mathbf{U}(1) \times \mathbf{S p}(n) \times \mathbf{U}(1)$ & $\mu_{1} \otimes \nu_{n} \oplus \nu_{n} \otimes \mu_{1}$ & 4 & $n \geq 2$ \\
$9 a$ & $\mathbf{S p}(1) \times \mathbf{S p}(n) \times \mathbf{S p}(1)$ & $\nu_{1} \otimes \nu_{n} \oplus \nu_{n} \otimes \nu_{1}$ & 3 & $n \geq 2$ \\
$9 b$ & $\mathbf{U}(1) \times \mathbf{S p}(n) \times \mathbf{S p}(1)$ & $\mu_{1} \otimes \nu_{n} \oplus \nu_{n} \otimes \nu_{1}$ & 3 & $n \geq 2$ \\
$9 c$ & $\mathbf{S p}(n) \times \mathbf{S p}(1)$ & $\nu_{n} \otimes \nu_{1} \oplus \nu_{n}$ & 3 & $n \geq 2$ \\
10 & $\mathbf{S p}(n) \times \mathbf{S p}(1)$ & $\nu_{n} \otimes \nu_{1} \oplus \nu_{n} \otimes \nu_{1}$ & 4 & $n \geq 2$ \\
\hline
\end{tabular}

TABle 2: GoOd ORBIFOLD QUOTIENTS

\begin{tabular}{|c|c|c|c|c|}
\hline Case & $G$ & $\rho$ & Cohom & Conditions \\
\hline 11 & $\mathbf{U}(1)$ & $\left(\mu_{1}\right)^{r} \oplus\left(\mu_{1}\right)^{s}$ & 3 & $r \neq s, r s \neq 0$ \\
12 & $\mathbf{S U}(2) \times \mathbf{U}(1)$ & $\mu_{2} \otimes \mu_{1} \oplus \rho_{3}$ & 3 & - \\
$13 a$ & $\mathbf{S p}(2) \times \mathbf{S p}(1)$ & $\nu_{2} \otimes \nu_{1} \oplus \rho_{5}$ & 3 & - \\
$13 b$ & $\mathbf{S p}(2) \times \mathbf{U}(1)$ & $\nu_{2} \otimes \mu_{1} \oplus \rho_{5}$ & 3 & - \\
$13 c$ & $\mathbf{S p}(2)$ & $\nu_{2} \oplus \rho_{5}$ & 3 & - \\
14 & $\mathbf{S p i n}(9)$ & $\Delta_{9} \oplus \rho_{9}$ & 3 & - \\
\hline
\end{tabular}

TABLE 3: BAD ORBIFOLD QUOTIENTS

Proof. The representation $\rho=\rho_{1} \oplus \rho_{2}$, with $\rho_{i}$ being of cohomogeneity 1 , is polar if and only if it has cohomogeneity 2. This happens if and only if $\rho$ is orbit equivalent to the outer direct sum of the representations $\rho_{1}$ and $\rho_{2}$. In particular, it is the case if $G$ splits as $G=G_{1} \cdot G_{2}$, such that the restriction of $\rho_{i}$ to $G_{i}$ acts on $V_{i}$ with cohomogeneity 1 , for $i=1,2$.

The list of representations of cohomogeneity one is well known (see e.g. [GL14, §12.7]). Cases 1-4, 6, 7 and 10 correspond to a doubling representation. The circle group yields case 11 (and 1a).

Assume now that $G$ is not a circle and $\rho$ not a doubling representation. If $G$ is simple, then $\rho_{1}$ and $\rho_{2}$ must be two inequivalent representations of cohomogeneity one. We get cases $13 \mathrm{c}$ and 14; the remaining cases are representations of $\mathbf{S U}(4), \mathbf{S p i n}(7)$ and $\mathbf{S p i n}(8)$ of cohomogeneity 2 (cf. [Str96, p.84]) and one additional representation $\left(\mathbf{S U}(2), \mu_{2} \oplus \rho_{3}\right)$. The latter representation has a non-polar slice at $p \in \mathbb{R}^{3}$ given by $\left(\mathbf{U}(1), \mu_{1} \oplus \mu_{1} \oplus \theta\right)$, where $\theta$ denotes a trivial summand.

Otherwise two factors of $G$ act effectively on one summand, which we assume to be $V_{1}$. The first case is $\left(G / \operatorname{ker} \rho_{1}, V_{1}\right)=\left(\mathbf{U}(n), \mu_{n}\right), n \geq 2$. Since $\mathbf{S U}(n)$ acts with cohomogeneity 1 on $V_{1}$, the product of the complementary factors of $\mathbf{S U}(n)$ in $G$ does not act with cohomogeneity 
1 on $V_{2}$ (due to the observation in the beginning of the proof). We get cases 5 a and 12 , the representation $\left(\mathbf{U}(4), \mu_{4} \oplus \rho_{6}\right)$, which is polar, and the following candidates:

\begin{tabular}{|c|c|c|c|}
\hline$G$ & $\rho$ & Cohom & Conditions \\
\hline $\mathbf{U}(1) \times \mathbf{S U}(n)$ & $\left(\mu_{1}\right)^{r} \otimes \mu_{n} \oplus\left(\mu_{1}\right)^{s} \otimes \mu_{n}$ & 4 & $n=2$ or $r=s$ \\
$\mathbf{U}(1) \times \mathbf{S U}(n)$ & $\left(\mu_{1}\right)^{r} \otimes \mu_{n} \oplus\left(\mu_{1}\right)^{s} \otimes \mu_{n}$ & 3 & $n \geq 3$ and $r \neq s$ \\
\hline
\end{tabular}

Since $r=s$ is a doubling representation, for the first representation we may assume $n=2$; taking a slice representation at a point $p \in V_{1}$ shows that $\rho$ can be infinitesimally polar if and only if $r= \pm s$; as real representations, the two cases are equivalent one to the other and, after dividing by a finite ineffective group, we may assume $r=s=1$. The second representation yields case $5 \mathrm{~b}$.

The second case is $\left(G / \operatorname{ker} \rho_{1}, V_{1}\right)=\left(\mathbf{S p}(n) \times \mathbf{U}(1), \nu_{n} \otimes \mu_{1}\right), n \geq 2$. Again, the product of the complementary factors of $\mathbf{S p}(n)$ in $G$ does not act with cohomogeneity 1 on $V_{2}$. We get cases $8,9 \mathrm{~b}$ and $13 \mathrm{~b}$ plus $\left(\mathbf{U}(1) \times \mathbf{S p}(n),\left(\mu_{1}\right)^{r} \otimes \nu_{n} \oplus\left(\mu_{1}\right)^{s} \otimes \nu_{n}\right)$, where $n \geq 2$. This representation has cohomogeneity 5. Taking a slice representation shows that it can be infinitesimally polar if and only if $r= \pm s$; as real representations, the two cases are equivalent one to the other.

The last case is $\left(G / \operatorname{ker} \rho_{1}, V_{1}\right)=\left(\mathbf{S p}(n) \times \mathbf{S p}(1), \nu_{n} \otimes \nu_{1}\right), n \geq 1$. Again, the product of the complementary factors of $\mathbf{S p}(n)$ in $G$ does not act with cohomogeneity 1 on $V_{2}$. We get $\left(\mathbf{S p}(1) \times \mathbf{S p}(1), \nu_{1} \otimes \nu_{1} \oplus \rho_{3}\right)$, which is polar, plus cases 9a, 9b, 9c and 13a.

\section{MAIN STEP}

We are going to prove by induction on $k$ the following statement.

Theorem 7.1. Let $\rho: G \rightarrow \mathbf{S O}(n+1)$ be a non-polar representation of a compact Lie group $G$. Assume that $X^{k}=\mathbb{S}^{n} / G$ is a good Riemannian orbifold whose universal orbicovering is diffeomorphic to a sphere. Then $X^{k}$ has constant curvature 4 . Moreover, if $k \geq 3$, then the restriction of $\rho$ to the identity component of $G$ is the sum of two representations of cohomogeneity 1 . Finally, $k \leq 5$.

Proof. We proceed by induction on $k$. The case $k=1$ cannot occur, since such an action would be polar. The case $k=2$ is well known (Subsection 3.3 ). Assume now that $k \geq 3$, and that the result is true for all $k^{\prime}<k$. By considering the restriction of the action to the identity component of $G$, it suffices to prove the result for connected groups.

Either we have the Hopf action of $\mathbf{S p}(1)$ on $\mathbb{S}^{7}$, for which case all claims are true, or the quotient has boundary (Lemma 2.1 and Subsection [3.2). Thus we may assume $\partial X \neq \emptyset$. Due to Corollary [5.3, we find an isometric non-polar action of some compact Lie group $N$ on some unit sphere $\mathbb{S}^{m}$, such that the quotient $Y=\mathbb{S}^{m} / N$ is a good Riemannian orbifold of dimension $k-1$, whose universal orbi-covering is diffeomorphic to a sphere. By our inductive assumption, we deduce $k-1 \leq 5$. Hence $k \leq 6$. By the inductive assumption, $Y$ has constant curvature 4 , hence its universal orbi-covering $Z$ is a sphere of constant curvature 4 .

We first assume that $\rho$ is reducible. Due to Proposition 6.1 and Section 3 we only need to prove that $\mathbb{R}^{n+1}$ is the sum of two summands on each of which $G$ acts with cohomogeneity one, since this would imply that the quotient has curvature 4 and dimension $\leq 5$. Thus it suffices to prove that $G$ acts transitively on any non-trivial invariant great subsphere of $\mathbb{S}^{n}$. 
Let $\tilde{X}$ denote the universal orbi-covering of $X$. Then $\tilde{X}$ is a sphere with curvature $\geq 1$. By Corollary 5.3, it admits a reflection $r: \tilde{X} \rightarrow \tilde{X}$, whose set of fixed points $Z$ is a $(k-1)$ dimensional sphere of constant curvature 4 .

We claim that all geodesics in $\tilde{X}$ are closed of period $\pi$ (cf. [Wil01, Section 2.1]). Indeed, the element $-I d \in O(n+1)$ commutes with $G$ and induces an isometry $I$ on $X=\mathbb{S}^{n} / G$, which sends a point $p$ to the point $\exp (\pi h)$, where exp is the exponential map of the orbifold $X$ and $h$ is any unit vector at $p$. Hence, the map $\tilde{I}: \tilde{X} \rightarrow \tilde{X}$ which sends a point $p$ to the endpoint of any geodesic of length $\pi$ starting at $p$ is well-defined, and it is an isometry of $\tilde{X}$. Note that $\tilde{I}^{2}$ is the identity, and that $\tilde{I}$ sends any geodesic to itself without changing the orientation of the geodesic. All geodesics in $Z$ are closed of period $\pi$, thus the map $\tilde{I}$ fixes all points in $Z$. Hence $\tilde{I}$ is the identity or the reflection $r$. But $r$ changes the orientation on any geodesic intersecting $Z$ orthogonally. Hence $\tilde{I}$ is the identity. Thus all geodesics in $\tilde{X}$ are closed of period $\pi$.

In particular, the diameter of $\tilde{X}$ is at most $\frac{\pi}{2}$. For any point $p \in \tilde{X}$, we denote by $\operatorname{Ant}(p)$ the set of all points $q \in \tilde{X}$ with $d(p, q) \geq \frac{\pi}{2}$. We claim that for any point $p \in \tilde{X}$, the set Ant $(p)$ has at most one point.

To prove the claim, note first that the set $\operatorname{Ant}(p)$ contains with any pair of points $q_{1}, q_{2}$ any (non necessarily minimizing) geodesic of length $<\pi$ between these points by Toponogov's theorem. Since geodesics in $\tilde{X}$ are closed of period $\pi$, we see that $A n t(p)$ contains any closed geodesic going through any pair of its different points, hence $\operatorname{Ant}(p)$ is a totally geodesic submanifold of $\tilde{X}$. Therefore, if $\operatorname{Ant}(p)$ intersects $Z$ in at least 2 points, it must contain $Z$. Hence $p \notin Z$ and the distance of $p$ to $r(p)$ is larger than $\frac{\pi}{2}$, since $Z$ separates $\tilde{X}$. This contradicts the diameter bound.

Thus $Z$ intersects $\operatorname{Ant}(p)$ in at most one point. Assume that $A n t(p)$ contains at least two points, hence at least one closed geodesic $\gamma$. Now, the distance function to the subset $Z$ restricted to any of the two open subspaces $\tilde{X}^{ \pm}$in which $Z$ divides $\tilde{X}$ is strictly concave. Hence the closed geodesic $\gamma$ cannot be contained in any of this half-spaces of $\tilde{X}$. Thus, $\gamma$ intersects $Z$ in some point $q$. But by topological reasons, $\gamma$ cannot intersect $Z$ only at one point $q$. This contradiction proves the claim that $\operatorname{Ant}(p)$ consists of at most one point.

Let now $S=\mathbb{S}^{l}, 0 \leq l<n$ be a $G$-invariant great sphere in $\mathbb{S}^{n}$ and let $S^{\perp}$ be the orthogonal complement of $S$. Let $\bar{S}$ and $\bar{S}^{\perp}$ be the projections of these great spheres to $X$. Then for any point $p \in \bar{S}$ and any point $q \in \overline{S^{\perp}}$, we have $d(p, q)=\frac{\pi}{2}$. Since the orbi-covering $\tilde{X} \rightarrow X$ is 1-Lipschitz, we deduce that the sets $\bar{S}$ and $\overline{S^{\perp}}$ have only one element each (otherwise we would get in $\tilde{X}$ subset of the form $\operatorname{Ant}(p)$ or $\operatorname{Ant}(q)$ with more than one point). But this is exactly the statement that $G$ acts transitively on $S$ and on $S^{\perp}$.

It remains only to prove that $\rho$ cannot be irreducible. Suppose, to the contrary, $\rho$ is an irreducible non-polar representation of cohomogeneity $4 \leq k+1 \leq 7$ on $V=\mathbb{R}^{n+1}$. In view of Lemma 2.2 and the subsequent lines, we may replace this action by the identity component of its minimal reduction and assume that it has trivial principal isotropy groups. Due to Lemmas 2.4 and 2.3, all isotropy groups are connected and products of groups of rank one. In the cases $k=3$, 4, we refer to Tables 1 and 2 in [GL14, in which all irreducible representations of cohomogeneity 4 and 5 are classified, and check that each representation in the tables is not infinitesimally polar. Indeed, we need only check representations with trivial copolarity (i.e., those equal to their minimal reductions) and non-empty boundary 
which leaves us with the representation of $\mathbf{S O}(3) \times \mathbf{U}(2)$ only, which is not infinitesimally polar by [GL14, §8].

Now we may assume $k=5$ or 6 . In the case $G$ is simple, we refer to the table of non-polar representations of cohomogeneity 6 or 7 in [GL14, §12.8]. We exclude the representations of complex type by noting that they have toric reductions and thus are not infinitesimally polar (cf. Subsection 2.6). We exclude $\left(\mathbf{S U}(2), \mathbb{C}^{4}\right)$ by noting that its orbit space has empty boundary (it produces the smallest weighted quaternionic projective space, namely, the Hitchin orbifold $\mathrm{O}_{3}$ ), and the remaining representations of quaternionic type by noting that they have an isotropy group (corresponding to a highest weight vector) containing a simple factor of rank bigger than one. Finally, the remaining representation of $\mathbf{S O}(3)$ is also excluded because the slice representation at a 0 -weight vector is non-polar. Thus we may assume in addition that $G$ is non-simple.

To finish the proof, let $p \in \mathbb{S}^{n}$ be such that $\operatorname{rank}\left(G_{p}\right) \geq \operatorname{rank}(G)-1$ (cf. Wil03, Lemma 6.1]). The triviality of principal isotropy groups implies that slice representations are effective. Due to Lemmas 2.3 and 2.4, $G_{p}$ is a product of groups of rank one and there exists a normal simple factor $H$ of $G_{p}, H \cong \mathbf{U}(1)$ or $H \cong \mathbf{S p}(1)$, corresponding to a boundary stratum $S t$ of $X$. Moreover, we can choose $H \cong \mathbf{S p}(1)$, unless $G_{p}$ is a torus. According to Corollary 5.3 and its proof, there exists a non-polar isometric action of a compact connected Lie group $(N / H)^{0}$ on some unit sphere $\mathbb{S}^{m}$ such that the quotient $Y$ is a good Riemannian orbifold of dimension $k-1$ and its universal orbi-covering is diffeomorphic to a sphere, where $N$ is the normalizer of $H$ in $G$. By induction, we know that the action of $(N / H)^{0}$ on $W=\mathbb{R}^{m+1}$ is reducible and thus listed in Table 2 or 3 in Section 6. Using the fact that $k=5$ or 6 we deduce that $(N / H)^{0}$ is $\mathbf{S U}(2)(k=5)$ or $\mathbf{S p}(q)(k=6)$ or $\mathbf{S p}(q) \times \mathbf{U}(1)$ $(k=5)$ with their respective doubling representations. Since the isotropy groups of the action of $(N / H)^{0}$ on $\mathbb{S}^{m}$ are still products of groups of rank 1, in the latter two cases we get $q=2$.

By the choice of $H$, the group $G_{p}$ is contained in $N$. Then $G_{p} / H$ is the isotropy group of $(N / H, W)$ at $p \in W$. By the explicit form of this representation, we have $\operatorname{rank}\left(G_{p} / H\right)<$ $\operatorname{rank}(N / H)$ so $\operatorname{rank}\left(G_{p}\right) \leq \operatorname{rank}(N)-1$. Therefore $\operatorname{rank}(G)=\operatorname{rank}(N)$. Note also that $N \neq G$. Next we examine each case separately.

If $(N / H)^{0}=\mathbf{S U}(2)$ then $\operatorname{rank}(G)=2$. The representation $\left(\mathbf{S U}(2), \mathbb{C}^{2} \oplus \mathbb{C}^{2}\right)$ has all isotropy groups trivial, so $G_{p}=H$. Since $G$ is not simple and not equal to $N$, we deduce $H=\mathbf{U}(1)$ and $G$ is locally isomorphic to $\mathbf{S U}(2) \times \mathbf{S U}(2)$. Since the cohomogeneity of $\rho$ is 6 , we have $\operatorname{dim} V=12$. We are lead to a contradiction by [GL14, §10.5] which says that this representation has empty boundary.

In both the remaining cases, we see that the action $(N / H)^{0}$ on $W$ has a non-Abelian isotropy group. More precisely, there is a point $q \in W$, such that the isotropy group $K=$ $\left((N / H)^{0}\right)_{q}$ has a non-Abelian connected component $K^{0}$ with $\operatorname{rank}\left(K^{0}\right)=\operatorname{rank}(N / H)^{0}-1$. Thus the isotropy group $G_{q}$ is non-Abelian as well and satisfies $\operatorname{rank}\left(G_{q}\right) \geq \operatorname{rank}\left(N_{q}\right) \geq$ $\operatorname{rank}(N)-1=\operatorname{rank}(G)-1$. Thus we may replace $p$ by $q$ and, applying Lemma 2.3, assume that $H$ is isomorphic to $\mathbf{S p}(1)$.

If $(N / H)^{0}=\mathbf{S p}(2)$ then $\operatorname{rank}(G)=3$. The group $G$ is non-simple, and contains a subgroup locally isomorphic to $H \times \mathbf{S p}(2) \cong \mathbf{S p}(1) \times \mathbf{S p}(2)$. We deduce that $G$ is finitely covered by $\tilde{G}=\mathbf{S p}(1) \times \mathbf{S p}(2)$ and $H \cong \mathbf{S p}(1)$ is a normal subgroup of $G$, which contradicts the fact that $N \neq G$. 
If $(N / H)^{0}=\mathbf{S p}(2) \times \mathbf{U}(1)$ then $\operatorname{rank}(G)=4$. We see that $G$ contains a subgroup locally isomorphic to $H \times \mathbf{S p}(2) \times \mathbf{U}(1)$, with $H \cong \mathbf{S p}(1)$. Moreover, $H$ is not a normal subgroup of $G$, since $G \neq N$.

If $G$ has no simple factor of rank 3 , then it is locally isomorphic to $\operatorname{Sp}(2) \times G^{\prime}$, where $G$ and $N$ have a common $\mathbf{S p}(2)$-factor; since $W=\mathbb{H}^{2} \oplus \mathbb{H}^{2}$ is a subspace of $V$ invariant under this factor, we deduce that $V=\mathbb{H}^{2} \otimes_{\mathbb{F}} V^{\prime}$ where $V^{\prime}$ is a representation of $G^{\prime}$ and $\mathbb{F}=\mathbb{R}, \mathbb{C}$ or $\mathbb{H}$ according to whether $V^{\prime}$ is of real, complex or quaternionic type. In any case, $\operatorname{dim}_{\mathbb{R}} V$ is divisible by 8 . It follows that $\operatorname{dim} G^{\prime}=\operatorname{dim} V^{\prime}-\operatorname{dim} \operatorname{Sp}(2)-6$ is also divisible by 8 . Since $G^{\prime}$ has rank 2, we deduce that $G^{\prime}=\mathbf{S U}(3)$. Now $\operatorname{dim} V=\operatorname{dim} \mathbf{S p}(2) \times \mathbf{S U}(3)+6=24$, so $(G, V)=\left(\mathbf{S p}(2) \times \mathbf{S U}(3), \mathbb{C}^{4} \otimes_{\mathbb{C}} \mathbb{C}^{3}\right)$ and $H$ must be an $\mathbf{S U}(2)$-subgroup of $\mathbf{S U}(3)$. The fixed point set $Z$ of any such subgroup on $\mathbb{C}^{3}$ has complex dimension 1 , so the set of fixed points of $H$ in $\mathbb{C}^{4} \otimes_{\mathbb{C}} \mathbb{C}^{3}$ is $\mathbb{C}^{4} \otimes_{\mathbb{C}} Z$. Hence its complex dimension is not 8 , a contradiction.

Suppose next $G$ has a simple factor $G^{\prime}$ of rank 3. Then $G^{\prime}$ contains $\mathbf{S p}(2)$ and an $\mathbf{S p}(1)$ subgroup normalizing it. It follows that $G^{\prime}=\mathbf{S p}(3)$.

Now $G=\mathbf{S p}(3) \times \mathbf{S p}(1)$ and $\operatorname{dim} V=\operatorname{dim} G+6=30$ or $G=\mathbf{S p}(3) \times \mathbf{U}(1)$ and $\operatorname{dim} V=\operatorname{dim} G+6=28$. Since 30 is not divisible by 4 , in the first case $V$ is a real tensor product of irreducible representations of real type of $\mathbf{S p}(3)$ and $\mathbf{S p}(1)$. The irreducible representations of $\mathbf{S p}(3)$ of lowest degree (i.e. complex dimension) are the fundamental representations (cf. [Oni62, Lemma 3.1]) with degrees 6, 14 and 14, and are respectively of quaternionic, real, quaternionic type. Since 30 is not divisible by $14, G=\mathbf{S p}(3) \times \mathbf{S p}(1)$ is ruled out. On the other hand, in the second case we get two candidates $\left(\mathbf{S p}(3) \times \mathbf{U}(1), \Lambda^{2} \mathbb{C}^{6} \ominus \mathbb{C}\right)$ and $\left(\mathbf{S p}(3) \times \mathbf{U}(1), \Lambda^{3} \mathbb{C}^{6} \ominus \mathbb{C}^{6}\right)$. The former representation is excluded because the isotropy group at a point corresponding to (the projection along the trivial summand $\mathbb{C}$ of) a skewsymmetric bilinear form of rank 2 contains a rank 2 subgroup isomorphic to $\mathbf{S p}(2)$, and the latter one is excluded because the isotropy group at a highest weight vector contains a rank 2 subgroup isomorphic to $\mathbf{S U}(3)$; both situations contradict Lemma 2.3,

\section{Conclusion}

Now it is easy to deduce all the results stated in the introduction. Note that Theorem 1.5 has already been proved in Section 4.

Under the assumptions of Theorem 1.3, we see that the quotient $X$ has non-empty boundary (Lemma 2.1 and Subsection 3.2). By Theorem 1.5, $X$ is a good orbifold, whose universal orbi-covering is diffeomorphic to the sphere. Therefore, we may apply Theorem 7.1 to see that $\rho$ is the sum of two representations of cohomogeneity one. Now, Theorem 1.3 follows from Proposition 6.1 and the description of the quotients in Section 3 ,

Under the assumption of Theorem 1.4 we may assume that $G=G^{0}$. In all cases listed in the theorem, the quotient $X=\mathbb{S}^{n} / G$ is a Riemannian orbifold whose geometry is discussed in Section 3, On the other hand, assume that $X$ is a Riemannian orbifold of dimension at least 3 (so that $\rho$ has cohomogeneity at least 4 ) and that $\rho$ is not polar. If the quotient $X$ has no boundary then $\rho$ is almost free (Lemma 2.1) hence as stated (Subsection 3.2). If $X$ has boundary then $\rho$ satisfies the assumption of Theorem 7.1 (due to Theorem 1.5). Hence $\rho$ is one of the representations appearing in Proposition 6.1, in the only case in Table 2 of rank one, the quotient has no boundary, so this case cannot occur. It follows that $G$ has rank at least 2 and $\rho$ is as described in Theorem 1.3. This proves Theorem 1.4. 
Theorem 1.2 is a direct consequence of Theorem 1.4. Finally, to prove Theorem 1.1 we may again assume that $G$ is connected, since $\mathbb{S}^{n} / G$ and $\mathbb{S}^{n} / G^{0}$ have the same universal orbicovering. Thus we may apply Theorem 1.4. If $\rho$ is polar then $X$ has constant curvature 1 and the universal orbi-covering $\tilde{X}$ of $X$ is the sphere of constant curvature 1 . If $G$ has rank one and $X$ has empty boundary then $X$ is a weighted projective space as discussed in Subsection 3.2. Moreover, $X$ is simply connected as an orbifold in this case, hence $\tilde{X}=X$. If $X$ has dimension 2 and curvature $\neq 1$, then as proved by Straume [Str94], the quotient $X$ can be represented as the quotient $X=\mathbb{S}^{3} / G^{\prime}$, for some group $G^{\prime}$ of dimension 1 . Thus $X$ has as an orbi-covering $X^{\prime}=\mathbb{S}^{3} / \mathbf{U}(1)$, where $\mathbf{U}(1)$ acts without fixed points. In such a case $X^{\prime}$ is a weighted complex projective space, which is then the universal orbi-covering of $X$. Finally, if $X$ has constant curvature 4 as in Theorem 1.3 , then $\tilde{X}$ is a sphere of constant curvature 4.

\section{REFERENCES}

[AKLM03] D. Alekseevsky, A. Kriegl, M. Losik, and P. Michor, The Riemannian geometry of orbit spaces. metrics, geodesics, integrable systems, Publ. Math. Debrecen 62 (2003), 1-30.

[AKLM07] _ Reflection groups on Riemannian manifolds, Ann. Mat. Pura Appl. 186 (2007), 25-58.

[BCO03] J. Berndt, S. Console, and C. Olmos, Submanifolds and holonomy, Research Notes in Mathematics, no. 434, Chapman \& Hall/CRC, Boca Raton, 2003.

[Ber01] I. Bergmann, Reducible polar representations, Manuscripta Math. 104 (2001), no. 3, 309-324.

[Bes78] A. Besse, Manifolds all of whose geodesics are closed, Ergeb. Math. Ihrer Grenz., vol. 93, Springer, Berlin, 1978.

[BH99] M. Bridson and A. Haefliger, Metric spaces of non-positive curvature, Grundlehren der mathematischen Wissenschaften, no. 319, Springer Verlag, 1999.

[Dad85] J. Dadok, Polar coordinates induced by actions of compact Lie groups, Trans. Amer. Math. Soc. 288 (1985), 125-137.

[Dav10] M. Davis, Lectures on orbifolds and reflection groups, Higher Education Press, pp. 63-93, Springer-Verlag, 2010.

[Dea11] O. Dearricott, A 7-manifold with positive curvtaure, Duke. Math. J. 158 (2011), 307-346.

[EH99] J. Eschenburg and E. Heintze, On the classification of polar representations, Math. Z. 232 (1999), 391-398.

[FG14] F. Fang and K. Grove, Reflection groups in non-positive curvature, E-print arXiv:1403.5019 [math.DG], 2014.

[FGT12] F. Fang, K. Grove, and G. Thorbergsson, Tits geometry and positive curvature, E-print arXiv:1205.6222 [math.DG], 2012.

[GG88] D. Gromoll and K. Grove, The low-dimensional metric foliations of Euclidean spheres, J. Differential Geom. 28 (1988), 143-156.

[GL14] C. Gorodski and A. Lytchak, On orbit spaces of representations of compact Lie groups, J. reine angew. Math. 691 (2014), 61-100.

[Gor08] C. Gorodski, Taut reducible representations of compact simple Lie groups, Illinois J. Math. 52 (2008), 121-143.

[Gre00] S. J. Greenwald, Diameters of spherical Alexandrov spaces and curvature one orbifolds, Indiana Univ. Math. J. 49 (2000), no. 4, 1449-1479.

[GS00] K. Grove and C. Searle, Global G-manifold reductions and resolutions, Ann. Global. Anal. Geom. 18 (2000), 437-446.

[GUW09] V. Guillemin, A. Uribe, and Z. Wang, Geodesics on weighted projective spaces, Ann. Global Anal. Geom. 36 (2009), 205-220.

[GVZ11] K. Grove, L. Verdiani, and W. Ziller, An exotic $T^{1} S^{4}$ with positive curvature, Geom. Funct. Anal. 21 (2011), 499-524. 
[How95] R. Howe, Perspectives on invariant theory: Schur duality, multiplicity-free actions and beyond, The Schur lectures (1992) (Tel Aviv), Israel Math. Conf. Proc., vol. 8, Bar-Ilan Univ., Ramat Gan, 1995, pp. 1-182.

[HW09] F. Hang and X. Wang, Rigidity theorems for compact manifolds with positive Ricci curvature, J. Geom. Anal. 19 (2009), 628-642.

[KL11] B. Kleiner and J. Lott, Geometrization of three-dimensional orbifolds via Ricci flow, arXiv:1101.3733, 2011.

[LR79] D. Luna and R. W. Richardson, A generalization of the Chevalley restriction theorem, Duke Math. J. 46 (1979), 487-496.

[LT10] A. Lytchak and G. Thorbergsson, Curvature explosion in quotients and applications, J. Differential Geom. 85 (2010), 117-140.

[Lun75] D. Luna, Adhérence d'orbite et invariants, Invent. Math. 29 (1975), 231-238.

[LW13] A. Lytchak and B. Wilking, Riemannian foliations of spheres, E-print arXiv:1309.7884 [math.DG], 2013.

[Lyt10] A. Lytchak, Geometric resolution of singular Riemannian foliations, Geom. Dedicata 149 (2010), 379-395.

[MS05] J. McGowan and C. Searle, How tightly can you fold a sphere?, Diff. Geometry and Appl. 22 (2005), 81-104.

[Oni62] A. L. Oniščik, Inclusion relations between transitive compact transformation groups (Russian), Trudy Moskov. Mat. Obšč. 11 (1962), 199-242, English transl., Amer. Math. Soc. Transl. (2) 50 (1966), 5-58.

[Pet98] A. Petrunin, Parallel transportation for Alexandrov space with curvature bounded below, Geom. Funct. Anal. 8 (1998), 123-138.

[Pet07] _ Semiconcave functions in Alexandrov's geometry, Survey. Differ. Geom. 11 (2007), 137201.

[PT88] R. S. Palais and C.-L. Terng, Critical point theory and submanifold geometry, Lect. Notes in Math., no. 1353, Springer-Verlag, 1988.

[Rad12] M. Radeschi, Low dimensional singular Riemannian foliations in spheres, E-print arXiv:1203.6113 [math.DG], 2012.

[Rad14] C Clifford algebras and new singular Riemannian foliations in spheres, E-print arXiv:1401.2546v2, 2014.

[Sch80] G. W. Schwarz, Lifting smooth homotopies of orbit spaces, I.H.E.S. Publ. in Math. 51 (1980), $37-135$.

[Str94] E. Straume, On the invariant theory and geometry of compact linear groups of cohomogeneity $\leq 3$, Diff. Geom. and its Appl. 4 (1994), 1-23.

[Str96] Compact connected Lie transformation groups on spheres with low cohomogeneity, I, Mem. Amer. Math. Soc., no. 569, American Mathematical Society, 1996.

[Tho91] G. Thorbergsson, Isoparametric foliations and their buildings, Ann. of Math. (2) 133 (1991), 429-446.

[Tho00] _ A survey on isoparametric hypersurfaces and their generalizations, Handbook of Differential Geometry, vol. I, ch. 10, Elsevier Science, 2000.

[TT97] C.-L. Terng and G. Thorbergsson, Taut immersions into complete Riemannian manifolds, Tight and Taut Submanifolds (T. E. Ryan and S.-S. Chern, eds.), Math. Sci. Res. Inst. Publ. 32, Cambridge University Press, 1997, pp. 181-228.

[Wie14] S. Wiesendorf, Taut submanifolds and foliations, J. Differential Geom. 96 (2014), no. 3, 457-505.

[Wil01] B. Wilking, Index parity of closed geodesics and rigidity of Hopf fibrations, Invent. Math. 148 (2001), 281-295.

[Wil03] , Torus actions on manifolds of positive sectional curvature, Acta Math. 191 (2003), 259-297.

[Wil06] _ Positively curved manifolds with symmetry, Ann. of Math. 163 (2006), 607-668.

[Wol84] J.A. Wolf, Spaces of constant curvature, 5th ed., Publish or Perish, Houston, 1984.

[Zil07] W. Ziller, On the geometry of cohomogeneity one manifolds with positive curvature, arXiv:0707.3345, 2007.

[Zil12] _ Riemannian manifolds with positive sectional curvature, arXiv:1210.4102, 2012. 
Instituto de Matemática e Estatística, Universidade de São Paulo, Rua do Matão, 1010, SÃo PAUlo, SP 05508-090, Brazil

E-mail address: gorodski@ime.usp.br

Mathematisches Institut, Universität Zu Köln, Weyertal 86-90, 50931 Köln, Germany

E-mail address: alytchak@math.uni-koeln.de 